\title{
Predicting Fatigue Damage in Composites: A Bayesian Framework
}

\author{
Manuel Chiachío ${ }^{\mathrm{a}, *}$, Juan Chiachío ${ }^{\mathrm{a}}$, Guillermo Rus ${ }^{\mathrm{a}}$, James L. Beck ${ }^{\mathrm{b}}$ \\ ${ }^{a}$ Dept. Structural Mechanics and Hydraulic Engineering, University of Granada. \\ Campus de Fuentenueva s/n, 18071 Granada, Spain. \\ ${ }^{b}$ Division of Engineering and Applied Science, 9-94, California Institute of Technology, Pasadena, CA \\ 91125, USA.
}

\begin{abstract}
Modeling the progression of damage in composites materials is a challenge mainly due to the uncertainty in the multi-scale physics of the damage process and the large variability in behavior that is observed, even for tests of nominally identical specimens. As a result, there is much uncertainty related to the choice of the class of models among a set of possible candidates for predicting damage behavior. In this paper, a Bayesian prediction approach is presented to give a general way to incorporate modeling uncertainties for inference about the damage process. The overall procedure is demonstrated by an example with test data consisting of the evolution of damage in glass-fiber composite coupons subject to tensiontension fatigue loads. Results are presented for the posterior information about the model parameters together with the uncertainty associated with the model choice from a set of plausible fatigue models. This approach confers an efficient way to make inference for damage evolution using an optimum set of model parameters and, in general, to treat cumulative damage processes in composites in a robust sense.
\end{abstract}

Keywords: FRP composites, fatigue, Markov chains, Bayesian inverse problem, model class selection

*Corresponding author. e-mail: mchiachio@ugr.es Tel: (+34)958240037 Fax: (+34)958249959.

E.T.S Ingenieros de Caminos, Canales y Puertos Campus de Fuentenueva s/n, 18071, Granada, Spain

All the authors contributed equally to this work 


\section{Introduction}

In composite materials, fatigue damage represents one of the most important sources of uncertainty for in-service behavior. This leads to conservative designs and higher costs in manufacture and maintenance [1]. Throughout decades of investigation, numerous fatigue models have been proposed [2-5] and a large amount of data has been derived from expensive experimental programs. The vast majority of these models are deterministic semi-empirical formulations calibrated for a particular material configuration under some specific testing conditions. Therefore, they not only neglect the modeling uncertainty coming from the adoption of a single value for model parameters, but also from the selection of a particular model class (e.g., the parameterized mathematical structure of the model for the damage behavior).

Some researchers have addressed uncertainty in fatigue modeling using Bayesian methods, mostly focused on crack propagation in metals [6-11]. However the application of a full Bayesian inverse-problem framework for assessing the modeling uncertainty still remains very limited for the study of the fatigue degradation of composite materials, precisely where the benefits of this framework can be fully exploited to deal with the well-known uncertainty of the fatigue damage process.

In this work, a full Bayesian prediction and updating framework is presented in application to the problem of fatigue damage progression in composite materials. To this end, Bayes' Theorem is applied for two levels: first, to deal with the posterior information about the model parameters for a specific model class, and second, to assess a degree of plausibility of each model class within a candidate set of models [12]. Here, probability is interpreted as a multi-valued logic that expresses the degree of plausibility of a proposition conditioned on the given information, because this interpretation provides a rigorous foundation for the Bayesian approach $[13,14]$. Consequently, the approach has the advantage of being able to quantify the uncertainties associated with (1) model parameters and (2) model choice for the damage behavior, and then to further make robust response predictions of fatigue degradation that incorporate all the modeling uncertainty in a quantitative way. Figure 1 
provides a conceptual scheme of the proposed Bayesian framework for damage prediction.

A cumulative damage model based on the theory of Markov chains [15] is used to infer a complete damage process from batch sequences of damage data. The proposed framework is not limited to this model choice, but Markov chains damage models [16] are of major interest due to their versatility and efficiency. Markov chains were first applied to fatigue modeling in composites by Rowatt et al. [17] as an extension of the pioneering work of Bogdanoff et al. [16]. Other relevant examples of Markov chains models for fatigue in composites are found in [18-21] while for metals in [22-28]. More applications and theoretical insight about stochastic cumulative damage models are provided in [29-31]. As a novelty, a new model parameterization is introduced to account for the nonstationarity based on a generalization of the time transformation-condensation method first developed by Bogdanoff et al. [16].

As an example, the full Bayesian framework is applied to damage data for sixteen quasiisotropic open-hole S2-glass laminates subject to constant amplitude tension-tension fatigue loading. The results show that accounting for the underlying uncertainty in stochastic fatigue models allows one to obtain a more robust data-based model of the fatigue process, in the sense of a trade-off between data fitting and model complexity to avoid the extremes of over-fitting or under-fitting the data. For the same reason, this framework can be used to select a parsimonious number of parameters to represent the fatigue process degradation within the context of the stochastic model presented in this work.

Section 2 of this paper is devoted to providing the basis and main assumptions about the proposed Markov chain damage model and also to defining the stochastic model classes to be considered. In Section 3, the problem of inference of a Markov chain model is formulated using a Bayesian inverse problem framework. This section also gives the basis of Bayesian model class selection and the corresponding computational issues that must be addressed for our needs. In Section 4, our framework is applied to a set of fatigue data to serve as an example. Section 5 gives concluding remarks. 


\section{Forward prediction problem}

\subsection{Markov chain forward model}

The starting point of our fatigue modeling approach is to model the evolution of fatigue damage as a discrete-state Markov chain [15]. This approach models the damage as a nondecreasing stochastic process $\left\{x_{n}, n \in \mathbb{N}\right\}$ taking values in $\mathcal{X}=[0,1] \subset \mathbb{R}$, where $x_{n}$ at fatigue cycle $n$ is the macroscopic relative stiffness reduction at one specific point, section or element $[32,33]$. Damage passes monotonically through a finite set of damage states, $\mathcal{X}_{i} \subset \mathcal{X}$ where $i \in I=\{0, \ldots, s\} \subset \mathbb{N}$, until the "absorbing" state $\mathcal{X}_{s}$ is reached [16]. The no-damage state corresponds to $i=0$. Each damage state $\mathcal{X}_{i} \subset \mathcal{X}$ is a subinterval represented by its center $\bar{x}_{n}^{i}=2 i+1 / 2 s+2$ such that $p_{n}^{i} \triangleq p\left(\bar{x}_{n}^{i}\right) \triangleq P\left[x_{n} \in \mathcal{X}_{i}\right] \geqslant 0$. Once the damage at cycle $n$ is at a certain state $\bar{x}_{n}^{i}$, it may go only to the next state in one cycle $\bar{x}_{n+1}^{i+1}$ with probability $p_{n}^{i, i+1} \triangleq p\left(\bar{x}_{n+1}^{i+1} \mid \bar{x}_{n}^{i}\right)$, and remain in the same state with probability $p_{n}^{i, i} \triangleq p\left(\bar{x}_{n+1}^{i} \mid \bar{x}_{n}^{i}\right)=1-p_{n}^{i, i+1}$. Additionally, this approach assumes that the set of load cycles is divided into $\mathrm{N}$ disjoint subsets or "duty cycles" $(\mathrm{DC}), \mathcal{T}=\{1, \ldots, n, \ldots, N\} \subset \mathbb{N}$, in which damage can be accumulated.

From the last remarks it follows that the damage at a certain DC $n$ is entirely defined by means of the probabilities of all possible damage states $\left\{\bar{x}_{n}^{0}, \bar{x}_{n}^{1} \ldots, \bar{x}_{n}^{s}\right\}$, summarized by the probability mass function $(\mathrm{PMF}) \mathbf{p}_{n}=\left[p_{n}^{0}, p_{n}^{1}, \ldots, p_{n}^{s}\right]$. By Markov chain theory, the probability of damage at DC $n+1$ can be obtained as

$$
\mathbf{p}_{n+1}=\mathbf{p}_{n} \cdot \mathbf{P}_{n}
$$

with $\mathbf{P}_{n}$ the "one-step" probability transition matrix for DC $n[16]$, and for general $m>n$,

$$
\mathbf{p}_{m}=\mathbf{p}_{n} \cdot \prod_{j=n}^{m-1} \mathbf{P}_{j}
$$

For a certain DC $n$, the matrix $\mathbf{P}_{n}$ stores in its $(i+1, j+1)$ element the conditional probability of transition $p\left(\bar{x}_{n+1}^{j} \mid \bar{x}_{n}^{i}\right)=p_{n}^{i, j}$ from state $\bar{x}_{n}^{i}$ to $\bar{x}_{n+1}^{j}$ in one DC. The one-step assumption 
restricts the structure of $\mathbf{P}_{n}$ to a bi-diagonal $(s+1) \times(s+1)$ matrix with $p_{n}^{s, s}=1$, then:

$$
\mathbf{P}_{n}=\left(\begin{array}{ccccc}
p_{n}^{0,0} & p_{n}^{0,1} & & & \\
& p_{n}^{1,1} & p_{n}^{1,2} & & \\
& \ddots & \ddots & \\
& & p_{n}^{s-1, s-1} & p_{n}^{s-1, s} \\
& & & & 1
\end{array}\right)
$$

where

$$
p_{n}^{i, i}+p_{n}^{i, i+1}=1 ; \quad i \in\{0, \ldots, s-1\}
$$

If the transition probabilities $p_{n}^{i, j}$ do not depend on the duty cycle $n$, then $\mathbf{P}_{n}=\mathbf{P} \quad \forall n \in$ $\mathbb{N}$ and the process is termed stationary. Otherwise, in the more general case where the transition probabilities may change with time (i.e. with load cycles), the process is nonstationary. More details about Markov chain models of cumulative damage are given in [16].

\subsection{Parameterization for non-stationarity}

For the purpose of inference, a parameterization strategy is needed to avoid a high dimensional problem for characterizing the non-stationary Markov chain model described above. The strategy consists of adopting the same value of the one-step probability of transition along the process for every state $i \in\{0, \ldots, s-1\}$, except for the absorbing state in which $p_{n}^{s, s}=1$. This allows us to have a single probability transition matrix, now called $\mathbf{Q}$, that remains invariant during the process, defined as:

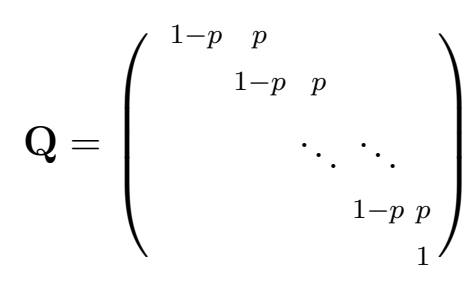

To account for the non-stationarity, an ad-hoc modification of the "natural" time scale $n$ into a transformed time scale $n^{\prime}=g^{\prime}(n)$ is introduced such that any probability transition matrix from step $n$ to step $m$ can be calculated as $\mathbf{Q}^{g^{\prime}(m)-g^{\prime}(n)}$, satisfying:

$$
\prod_{j=n}^{m-1} \mathbf{P}_{j}=\mathbf{Q}^{g^{\prime}(m)-g^{\prime}(n)} ; \quad m, n \in \mathbb{N}
$$


where $g^{\prime}(n)$ is a nonlinear function of $n$, that is given below. This procedure is based on the time transformation-condensation method (TTCM) first proposed by Bogdanoff and Kozin [16] by using undefined polynomials. Instead, we express $g^{\prime}(n)$ in terms of a continuous monotonic function $g(\theta):[0,1] \rightarrow[0,1]$ as follows:

$$
g^{\prime}(n)=N \cdot(g(n / N))
$$

where the function $g$ is suitably defined as an interpolating monotonic cubic spline [34] for a given set of $j \in \mathbb{N}$ interpolation points $\left\{\theta_{1}, \theta_{1}^{\prime}, \ldots, \theta_{j}, \theta_{j}^{\prime}\right\}$ and $N$ is a sufficiently large amount of duty cycles along which damage is completely developed. In this work, $N$ is chosen as the maximum length of the observed damage sequences. Notice also that to maintain the matrix structure of the model, the exponent $g^{\prime}(m)-g^{\prime}(n)$ in Equation 6 must be an integer after the transformation, to which an approximation to the nearest integer is applied, otherwise a condensation technique as proposed in [16] must be considered.

The transformation in Equation 7 distorts the natural time scale by using a nonlinear mapping over the unit interval, which has the double benefit over the TTCM of (1) having a bounded and defined searching space, and (2) keeping a fixed number of parameters. Figure 2 illustrates this concept. Each interpolation point is defined by its cartesian coordinates $\left[\theta_{j}, \theta_{j}^{\prime}\right]$ in the unit time scale, and together with $p$, act as model parameters for the Markov chain model that allows for a complete description of the fatigue damage process. Then the Markov chain damage model can be reformulated by replacing Equation 2 with:

$$
\mathbf{p}_{m}=\mathbf{p}_{n} \cdot \mathbf{Q}^{g^{\prime}(m)-g^{\prime}(n)}
$$

\subsection{Model class definition}

We denote by $\mathcal{M}_{j}$ the $j^{\text {th }}$ Bayesian model class [12] that incorporates the Markov chain forward model with $\boldsymbol{\theta}_{j}=\left\{\theta_{1}, \theta_{1}^{\prime}, \ldots, \theta_{j}, \theta_{j}^{\prime}, p\right\} \in \boldsymbol{\Theta}_{j}$ as model parameters along with the prior PDF $p\left(\boldsymbol{\theta}_{j} \mid \mathcal{M}_{j}\right)$, that gives the initial relative plausibility of each value of $\boldsymbol{\theta}_{j}$ before the information from measurements is incorporated. Henceforth, we drop the subscript $j$ on $\boldsymbol{\theta}$ since conditioning on $\mathcal{M}_{j}$ is sufficient to indicate which parameter vector is being considered. Based on this definition, a discrete set of possible candidate model classes 
$\mathbf{M}=\left\{\mathcal{M}_{j}, j: 1,2, \ldots, N_{M}\right\}, N_{M} \in \mathbb{N}$, is obtained. See Table 1 for a summary of the parameterization of the set of model classes $\mathbf{M}$.

\section{Bayesian inverse problem}

A rigorous foundation for the Bayesian approach to inverse problems is given by the CoxJaynes theory of probability as a multi-valued logic for plausible inference [14, 35, 36]. The focus in Bayesian inversion [37] is to investigate the posterior probability density function of the model parameters $\boldsymbol{\theta}$ over the set $\boldsymbol{\Theta} \subset \mathbb{R}^{d}$ of possible values, which is given by Bayes' Theorem. This PDF is interpreted as a measure of the relative plausibility of the values of $\boldsymbol{\theta}$ conditional on the available information. It is a measure of the uncertainty about the parameter values and not an inherent property of the real system. The available information is based on a set of data $\mathcal{D}$ from measurements on the system together with any relevant prior information that can be utilized.

We denote the likelihood function for model class $\mathcal{M}_{j} \in \mathbf{M}$ by $p\left(\mathcal{D} \mid \boldsymbol{\theta}, \mathcal{M}_{j}\right)$. It provides a measure of how well the model specified by $\boldsymbol{\theta}$ predicts the actual data $\mathcal{D}$. It is given by the PDF defined by the stochastic forward model parameterized by $\boldsymbol{\theta}$ when it is evaluated at data $\mathcal{D}$. Bayes' Theorem gives the posterior $\operatorname{PDF} p\left(\boldsymbol{\theta} \mid \mathcal{D}, \mathcal{M}_{j}\right)$ for the model specified by $\boldsymbol{\theta}$ in the class $\mathcal{M}_{j}$, as:

$$
p\left(\boldsymbol{\theta} \mid \mathcal{D}, \mathcal{M}_{j}\right)=c^{-1} p\left(\mathcal{D} \mid \boldsymbol{\theta}, \mathcal{M}_{j}\right) p\left(\boldsymbol{\theta} \mid \mathcal{M}_{j}\right)
$$

where $c$ is a normalizing constant, so that:

$$
\int_{\Theta} p\left(\boldsymbol{\theta} \mid \mathcal{D}, \mathcal{M}_{j}\right) d \boldsymbol{\theta}=c^{-1} \int_{\boldsymbol{\Theta}} p\left(\mathcal{D} \mid \boldsymbol{\theta}, \mathcal{M}_{j}\right) p\left(\boldsymbol{\theta} \mid \mathcal{M}_{j}\right) d \boldsymbol{\theta}=1
$$

Notice that Bayes' Theorem takes the initial quantification of the plausibility of each model specified by $\boldsymbol{\theta}$ in the model class $\mathcal{M}_{j}$, which is expressed by the prior probability distribution, and updates this plausibility by using the information in the data $\mathcal{D}$ expressed through the likelihood function. Note also that the normalizing constant $c$ in Bayes' Theorem does not affect the shape of the posterior distribution. The difficulty in applying Bayes' Theorem is that $c$ cannot usually be evaluated analytically nor it is readily calculated by numerical 
integration methods, if the dimension $d$ is not small. However, stochastic simulation based on MCMC methods can be used to obtain samples from the posterior without knowing $c$ in Equation 9, as done in Section 3.2.

\subsection{Formulation of the likelihood function}

In our problem, data $\mathcal{D}$ consist of a set of $K$ experimental sequences of fatigue-based damage from $K$ nominally-identical specimens, $\hat{\mathbf{Y}}=\left\{\hat{Y}^{(1)}, \ldots, \hat{Y}^{(k)}, \ldots, \hat{Y}^{(K)}\right\}$ where $\hat{Y}^{(k)}=$ $\left[\hat{y}_{n_{1}}^{(k)}, \hat{y}_{n_{2}}^{(k)}, \ldots, \hat{y}_{n_{N}}^{(k)}\right]$, is the measured relative stiffness reduction at a discrete set of regularly scheduled or even opportunistically staggered duty cycles $\mathcal{T}_{\mathcal{D}}=\left\{n_{1}, n_{2}, \ldots, n_{N}\right\}$, such that $\mathcal{T}_{\mathcal{D}} \subseteq \mathcal{T}$. A correspondence between the observed damage sequence $\hat{Y}^{(k)}=$ $\left[\hat{y}_{n_{1}}^{(k)}, \hat{y}_{n_{2}}^{(k)}, \ldots, \hat{y}_{n_{N}}^{(k)}\right]$ and the latent sequence of damage states $\left[\bar{x}_{n_{1}}^{i,(k)}, \bar{x}_{n_{2}}^{j,(k)}, \ldots, \bar{x}_{n_{N}}^{l,(k)}\right]$, with $i, j \ldots, l \in I$, can be established based on the defined set $\left\{\bar{x}_{n}^{0}, \bar{x}_{n}^{1}, \ldots, \bar{x}_{n}^{s}\right\}$ in Section 2.1, by taking $\bar{x}_{n}^{i,(k)}$ when $\hat{y}_{n}^{(k)} \in \mathcal{X}_{i}$. See Figure 3 for further details.

The likelihood function can be formulated as follows. First, the probability to observe a sequence of damage states $\left\{\bar{x}_{n_{1}}^{i,(k)}, \bar{x}_{n_{2}}^{j,(k)}, \ldots, \bar{x}_{n_{N}}^{l,(k)}\right\}$ in the $k^{\text {th }}$ specimen at the given set of duty cycles $\mathcal{T}_{\mathcal{D}}=\left\{n_{1}, n_{2}, \ldots, n_{N}\right\}, \mathcal{T}_{\mathcal{D}} \subseteq \mathcal{T}$, is modeled as a Markov chain parameterized by $\boldsymbol{\theta}$. By means of the Markov property, this probability can be obtained as follows:

$$
p\left(\bar{x}_{n_{1}}^{i}, \bar{x}_{n_{2}}^{j}, \ldots, \bar{x}_{n_{N}}^{l} \mid \boldsymbol{\theta}\right)=p\left(\bar{x}_{n_{1}}^{i} \mid \boldsymbol{\theta}\right) p\left(\bar{x}_{n_{2}}^{j} \mid \bar{x}_{n_{1}}^{i}, \boldsymbol{\theta}\right) \cdots p\left(\bar{x}_{n_{N}}^{l} \mid \bar{x}_{n_{N-1}}^{k}, \boldsymbol{\theta}\right)
$$

where the superscript $(k)$ denoting the $k^{\text {th }}$ specimen is not used in the last equation, and furthermore, the conditioning on the model class $\mathcal{M}_{j}$ is omitted. The likelihood function is then the product of probabilities as in Equation 11 over all $K$ specimen test sequences.

The structure of this likelihood function can be clarified by introducing the matrix $\mathbf{f}_{n_{h}}$, called the transition count matrix, that accounts for the number of observed transitions $\bar{x}_{n_{h}}^{i} \rightarrow \bar{x}_{n_{h+1}}^{j}$, i.e., the number of times for which the damage reaches the state $j$ at DC $n_{h+1}$, given that it previously was in state $i$ at $\mathrm{DC} n_{h}[38,39]$. In mathematical terms, the $(i, j)$ element of this matrix can be expressed as:

$$
f_{n_{h}}^{i, j}=\sum_{k=1}^{K} \sum_{i^{*}, j^{*}=0}^{s} \mathbb{I}\left(\bar{x}_{n_{h}}^{i^{*}=i,(k)}, \bar{x}_{n_{h+1}}^{j^{*}=j,(k)}\right)
$$


with $i^{*}, j^{*}=0, \ldots, s \in I$ and $\bar{x}_{n_{h}}^{i(k)}$ the damage state $i$ at duty cycle $n_{h}$, for the $k^{\text {th }}$ specimen. In the last expression, $\mathbb{I}\left(\bar{x}_{n_{h}}^{i^{*}=i,(k)}, \bar{x}_{n_{h+1} j^{*}=j,(k)}\right)$ is an indicator function which assigns the value of 1 when the transition $\bar{x}_{n_{h}}^{i} \rightarrow \bar{x}_{n_{h+1}}^{j}$ holds, and 0 otherwise. See Figure 3 for a detailed example about the construction of $\mathbf{f}_{n_{h}}$ considering two hypothetical curves of stiffness reduction from two specimens. The likelihood function can then be formulated as:

$$
p\left(\mathcal{D} \mid \boldsymbol{\theta}, \mathcal{M}_{j}\right)=p\left(\bar{x}_{n_{1}}^{i} \mid \boldsymbol{\theta}\right) \prod_{n_{h}=n_{1}}^{n_{N-1}} \prod_{i, j=0}^{s}\left(p\left(\bar{x}_{n_{h+1}}^{j} \mid \bar{x}_{n_{h}}^{i}, \boldsymbol{\theta}\right)\right)^{f_{n_{h}}^{i, j}}
$$

where $p\left(\bar{x}_{n_{h+1}}^{j} \mid \bar{x}_{n_{h}}^{i}, \boldsymbol{\theta}\right)$ is the probability transition between damage states $i$ and $j$ at DC $n_{h}$ and $n_{h+1}$ respectively, corresponding to the $(i+1, j+1)$ element of the matrix $\mathbf{Q}^{g^{\prime}\left(n_{h+1}\right)-g^{\prime}\left(n_{h}\right)}$, which is obtained by the model parameterization $\boldsymbol{\theta} \in \mathcal{M}_{j}$.

Finally, we remark that in constructing the likelihood function in Equation 13, any measurement error when assigning the $i$ th damage state based on the observed value $\hat{y}_{n} \in \mathcal{X}_{i}$ at duty cycle $n$ is subsumed by the uncertainty in the damage states described by the Markov chain model, and so it is not explicitly modeled.

\subsection{Stochastic simulation}

The goal of stochastic simulation methods in Bayesian updating is to generate samples which are distributed according to the posterior probability density function (PDF) in Equation 9. For this task, several algorithms have been proposed in the literature such as the Metropolis-Hastings, Gibbs Sampler and Hybrid Monte Carlo algorithms. A recent comprehensive overview of MCMC algorithms is given by Liang et al. [40].

Among them, the Metropolis-Hastings (M-H) algorithm is widely used for its versatility and implementation simplicity [41, 42]. This algorithm generates samples from a specially constructed Markov chain whose stationary distribution is any specified target PDF, known up to a scaling constant. By sampling a candidate vector $\boldsymbol{\theta}^{\prime}$ from a proposal distribution $q\left(\boldsymbol{\theta}^{\prime} \mid \boldsymbol{\theta}\right)$, the $\mathrm{M}-\mathrm{H}$ algorithm obtains the state of the chain at $\zeta+1$, given the state at $\zeta$, specified by $\boldsymbol{\theta}^{(\zeta)}$. The candidate $\boldsymbol{\theta}^{\prime}$ is accepted as the next state of the chain with probability $\min \{1, r\}$, where:

$$
r=\frac{p\left(\boldsymbol{\theta}^{\prime} \mid \mathcal{D}, \mathcal{M}\right) q\left(\boldsymbol{\theta}^{(\zeta)} \mid \boldsymbol{\theta}^{\prime}\right)}{p\left(\boldsymbol{\theta}^{(\zeta)} \mid \mathcal{D}, \mathcal{M}\right) q\left(\boldsymbol{\theta}^{\prime} \mid \boldsymbol{\theta}^{(\zeta)}\right)}
$$


If $\boldsymbol{\theta}^{\prime}$ is rejected, the previous state is repeated, $\boldsymbol{\theta}^{(\zeta+1)}=\boldsymbol{\theta}^{(\zeta)}$.

An important consideration is the specification of the variance $\sigma_{q}^{2}$ for the proposal distribution, which has a significant impact on the speed of convergence of the algorithm [42]. Small values tend to produce candidate samples that are accepted with high probabilities, but may result in highly dependent chains that explore the state space very slowly. In contrast, large values of $\sigma_{q}^{2}$ tend to produce large steps in state space, but result in small acceptance rates ${ }^{1}$. Thus, it is often worthwhile to select appropriate proposal variances by controlling the acceptance rates in a certain range, depending on the dimension $d$ of the proposal PDF, via some pilot runs $[43,44]$. The interval $[20 \%-40 \%]$ is suggested for the acceptance rate in low dimensional spaces, say $d \leqslant 10$, as in our case.

\subsection{Model-class assessment}

Following the model class definition given in Section 2.3, different model classes can be formulated and hypothesized to idealize the experimental system, and the previous Bayesian theory can be applied to each of them. Then, to select among a set of candidate model classes $\mathbf{M}=\left\{\mathcal{M}_{j}, j=1, \ldots, N_{M}\right\}$, a rigorous procedure is used to Bayesian model class assessment that judges the relative plausibility of each candidate model class based on their probabilities $p\left(\mathcal{M}_{j} \mid \mathcal{D}, \mathbf{M}\right)$ conditional on data $[12,45,46]$, where the probabilities can be obtained by Bayes' Theorem at the model class level:

$$
p\left(\mathcal{M}_{j} \mid \mathcal{D}, \mathbf{M}\right)=\frac{p\left(\mathcal{D} \mid \mathcal{M}_{j}\right) p\left(\mathcal{M}_{j} \mid \mathbf{M}\right)}{\sum_{i=1}^{N_{M}} p\left(\mathcal{D} \mid \mathcal{M}_{i}\right) p\left(\mathcal{M}_{i} \mid \mathbf{M}\right)}
$$

In the last equation, $p\left(\mathcal{M}_{j} \mid \mathbf{M}\right)$ is the prior probability of each $\mathcal{M}_{j}$, that expresses the user's judgement on the initial relative plausibility of $\mathcal{M}_{j}$. The factor $p\left(\mathcal{D} \mid \mathcal{M}_{j}\right)$ is called the evidence (or marginal likelihood) for the model class $\mathcal{M}_{j}$ provided by the observed data $\mathcal{D}$. It expresses how likely these data are according to the model class $\mathcal{M}_{j}$, and it can be obtained as follows:

$$
p\left(\mathcal{D} \mid \mathcal{M}_{j}\right)=\int_{\Theta} p\left(\mathcal{D} \mid \boldsymbol{\theta}, \mathcal{M}_{j}\right) p\left(\boldsymbol{\theta} \mid \mathcal{M}_{j}\right) \mathrm{d} \boldsymbol{\theta}
$$

\footnotetext{
${ }^{1}$ Defined as the relation between the number of accepted samples over the total amount of candidate samples.
} 
Notice also that the evidence is equal to the normalizing constant $c$ in establishing the posterior PDF in Equations 9 and 10.

Once the evidence $p\left(\mathcal{D} \mid \mathcal{M}_{j}\right)$ is computed for each model class, their values allow us to rank the model classes according to how plausible they are based on observations. In certain cases, more than one model class may have significant posterior probability in comparison with the rest of the set $\mathbf{M}$. Then, posterior model class averaging provides a coherent mechanism to account all these model classes for response prediction [12, 45, 47], as is shown in Section 4.

\subsubsection{Interpretation of model class evidence}

From the perspective of forward modeling problems, more complex models may be preferred over simpler models because they are considered more realistic. For inverse problems, however, this may lead to over-fitting of the data where the model is unnecessarily adjusted to fit the specific set of data used. Then the model does not generalize well when making predictions. The Bayesian approach to model class assessment shows that the posterior probability of each model class (or directly the evidence when $\left.p\left(\mathcal{M}_{j} \mid \mathbf{M}\right)=1 / N_{M}\right)$ automatically enforces a quantitative expression of a Principle of Model Parsimony or Ockham's razor $[14,48]$, by which simpler models that are reasonably consistent with data should be preferred over more complex models that lead to only slightly better agreement with the data. In the case of globally identifiable model classes $[49,50]$ based on the data, the posterior PDF in Equation 9 may be accurately approximated by a Gaussian distribution, and the evidence term can be obtained by Laplace's approximation [46, 49, 51].

In the more general case where the posterior PDF may not be approximated well by a Gaussian distribution, Muto and Beck [52] proposed an information theoretic point of view for the interpretation of the evidence for a model class, as follows:

$$
\log p\left(\mathcal{D} \mid \mathcal{M}_{j}\right)=\int_{\Theta}\left[\log p\left(\mathcal{D} \mid \boldsymbol{\theta}, \mathcal{M}_{j}\right)\right] p\left(\boldsymbol{\theta} \mid \mathcal{D}, \mathcal{M}_{j}\right) d \boldsymbol{\theta}-\int_{\Theta}\left[\log \frac{p\left(\boldsymbol{\theta} \mid \mathcal{D}, \mathcal{M}_{j}\right)}{p\left(\boldsymbol{\theta} \mid \mathcal{M}_{j}\right)}\right] p\left(\boldsymbol{\theta} \mid \mathcal{D}, \mathcal{M}_{j}\right) d \boldsymbol{\theta}
$$

This expression is obtained by strategically multiplying the logarithm of the evidence by a 
factor of one:

$$
\log p\left(\mathcal{D} \mid \mathcal{M}_{j}\right)=\left(\log p\left(\mathcal{D} \mid \mathcal{M}_{j}\right)\right) \underbrace{\int_{\boldsymbol{\Theta}} p\left(\boldsymbol{\theta} \mid \mathcal{D}, \mathcal{M}_{j}\right) d \boldsymbol{\theta}}_{=1}
$$

and then making substitutions according to Bayes' Theorem in Equation 9 to expand the evidence.

The first term of the right side of Equation 17 is the posterior mean of the log-likelihood function, which is a measure of the average goodness of fit of the model class $\mathcal{M}_{j}$ to the data $\mathcal{D}$. It accounts for the goodness of fit for different combinations of the model parameters, weighted by their posterior probabilities $[12,52]$. The second term is the relative entropy between the posterior and prior PDF of the model parameters. It can be interpreted as the expected information gain [EIG] about the model parameters from the data $\mathcal{D}$ and it will usually be larger for more complex models with more parameters. Applying Equation 15 therefore provides an automatic trade-off between model simplicity and fitting to observations, which explicitly reveals a quantitative Ockham's razor [12].

\subsubsection{Computation of the evidence for a model class}

The calculation of the evidence given in Equation 16 is not a trivial task. If the conditions cited in the last section for analytically approximating the posterior do not apply, or if the amount of data is small [46], then stochastic simulation methods are required. One straight-forward way to approximate the evidence is by considering the probability integral in Equation 16 as a mathematical expectation of the likelihood $p\left(\mathcal{D} \mid \boldsymbol{\theta}, \mathcal{M}_{j}\right)$ with respect to the prior $p\left(\boldsymbol{\theta} \mid \mathcal{M}_{j}\right)$. This approach leads to the direct Monte Carlo method as follows,

$$
p\left(\mathcal{D} \mid \mathcal{M}_{j}\right) \approx \frac{1}{N_{1}} \sum_{k=1}^{N_{1}} p\left(\mathcal{D} \mid \boldsymbol{\theta}^{(k)}, \mathcal{M}_{j}\right)
$$

where the $\boldsymbol{\theta}^{(k)}$ are $N_{1}$ samples drawn from the prior. Although this calculation can be easily implemented, it results in a computationally inefficient method (large-variance estimator), since the region of probability content of $p\left(\boldsymbol{\theta} \mid \mathcal{M}_{j}\right)$ is usually very different from the region where the likelihood $p\left(\mathcal{D} \mid \boldsymbol{\theta}, \mathcal{M}_{j}\right)$ has its largest values. To overcome this problem, some techniques for calculating the evidence based on samples from the posterior $p\left(\boldsymbol{\theta} \mid \mathcal{D}, \mathcal{M}_{j}\right)$ 
have received attention, although with known drawbacks of instability [53]. In this paper, a recent stable technique based on an analytical approximation of the posterior is used [54]. The relevant details from [54] are presented here in a concise way with special focus on the Metropolis-Hastings algorithm, which is the algorithm used in this work.

Let $K\left(\boldsymbol{\theta} \mid \boldsymbol{\theta}^{*}\right)$ be the transition PDF of any MCMC algorithm with stationary PDF $\pi(\boldsymbol{\theta})=$ $p\left(\boldsymbol{\theta} \mid \mathcal{D}, \mathcal{M}_{j}\right)$. The stationarity condition for the MCMC algorithm satisfies the following relation:

$$
\pi(\boldsymbol{\theta})=\int K\left(\boldsymbol{\theta} \mid \boldsymbol{\theta}^{*}\right) \pi\left(\boldsymbol{\theta}^{*}\right) d \boldsymbol{\theta}^{*}
$$

A general choice of $K\left(\boldsymbol{\theta} \mid \boldsymbol{\theta}^{*}\right)$ that applies to many MCMC algorithms, can be defined as:

$$
K\left(\boldsymbol{\theta} \mid \boldsymbol{\theta}^{*}\right)=T\left(\boldsymbol{\theta} \mid \boldsymbol{\theta}^{*}\right)+\left(1-a\left(\boldsymbol{\theta}^{*}\right)\right) \delta\left(\boldsymbol{\theta}-\boldsymbol{\theta}^{*}\right)
$$

where $T\left(\boldsymbol{\theta} \mid \boldsymbol{\theta}^{*}\right)$ is a smooth function that does not contain delta functions and $a\left(\boldsymbol{\theta}^{*}\right)$ is the acceptance probability which must satisfy $a\left(\boldsymbol{\theta}^{*}\right)=\int T\left(\boldsymbol{\theta} \mid \boldsymbol{\theta}^{*}\right) d \boldsymbol{\theta} \leqslant 1$. By substituting Equation 21 into 20, an analytical approximation of the posterior results as follows:

$$
\pi(\boldsymbol{\theta})=p\left(\boldsymbol{\theta} \mid \mathcal{D}, \mathcal{M}_{j}\right)=\frac{\int T\left(\boldsymbol{\theta} \mid \boldsymbol{\theta}^{*}\right) \pi\left(\boldsymbol{\theta}^{*}\right) d \boldsymbol{\theta}^{*}}{a(\boldsymbol{\theta})} \approx \frac{1}{a(\boldsymbol{\theta}) N_{1}} \sum_{k=1}^{N_{1}} T\left(\boldsymbol{\theta} \mid \boldsymbol{\theta}^{(k)}\right)
$$

where the $\boldsymbol{\theta}^{(k)}$ are $N_{1}$ samples distributed according to the posterior. For the special case of the Metropolis-Hastings algorithm, $T\left(\boldsymbol{\theta} \mid \boldsymbol{\theta}^{*}\right)=r\left(\boldsymbol{\theta} \mid \boldsymbol{\theta}^{*}\right) q\left(\boldsymbol{\theta} \mid \boldsymbol{\theta}^{*}\right)$, where $q\left(\boldsymbol{\theta} \mid \boldsymbol{\theta}^{*}\right)$ is the proposal $\mathrm{PDF}$, and $r\left(\boldsymbol{\theta} \mid \boldsymbol{\theta}^{*}\right)$ is given by:

$$
r\left(\boldsymbol{\theta} \mid \boldsymbol{\theta}^{*}\right)=\min \left\{1, \frac{p\left(\mathcal{D} \mid \boldsymbol{\theta}, \mathcal{M}_{j}\right) p\left(\boldsymbol{\theta} \mid \mathcal{M}_{j}\right) q\left(\boldsymbol{\theta}^{*} \mid \boldsymbol{\theta}\right)}{p\left(\mathcal{D} \mid \boldsymbol{\theta}^{*}, \mathcal{M}_{j}\right) p\left(\boldsymbol{\theta}^{*} \mid \mathcal{M}_{j}\right) q\left(\boldsymbol{\theta} \mid \boldsymbol{\theta}^{*}\right)}\right\}
$$

Additionally, for this algorithm, the denominator in Equation 22 can be approximated by an estimator that uses samples from the proposal distribution as follows:

$$
a(\boldsymbol{\theta})=\int r(\tilde{\boldsymbol{\theta}} \mid \boldsymbol{\theta}) q(\tilde{\boldsymbol{\theta}} \mid \boldsymbol{\theta}) d \tilde{\boldsymbol{\theta}} \approx \frac{1}{N_{2}} \sum_{k=1}^{N_{2}} r\left(\tilde{\boldsymbol{\theta}}^{(k)} \mid \boldsymbol{\theta}\right)
$$

where the $\tilde{\boldsymbol{\theta}}^{(k)}$ are $N_{2}$ samples from $q(\tilde{\boldsymbol{\theta}} \mid \boldsymbol{\theta})$, when $\boldsymbol{\theta}$ is fixed. Once the analytical approximation to the posterior in Equation 22 is set, then Equation 9 can be used to evaluate the 
evidence,

$$
\log p\left(\mathcal{D} \mid \mathcal{M}_{j}\right) \approx \log p\left(\mathcal{D} \mid \boldsymbol{\theta}, \mathcal{M}_{j}\right)+\log p\left(\boldsymbol{\theta} \mid \mathcal{M}_{j}\right)-\underbrace{\log p\left(\boldsymbol{\theta} \mid \mathcal{D}, \mathcal{M}_{j}\right)}_{\text {Analytical approx. }}
$$

Bayes' Theorem ensures that the last equation is valid for all $\boldsymbol{\theta} \in \boldsymbol{\Theta}$, so it is possible to use only one value for this parameter. However a more accurate estimate for the log-evidence can be obtained by averaging the results from Equation 25 using different values for $\boldsymbol{\theta}$ [54]. In this work, three different values of $\boldsymbol{\theta}$ from the peak region of $p(\boldsymbol{\theta} \mid \mathcal{D}, \mathcal{M})$ are employed for the evidence calculation. Once the evidence is obtained, the data-fit term in Equation 17 can also be estimated based on the $N_{1}$ samples from the posterior, and then the EIG term in this equation can be approximated by:

$$
\mathbb{E}\left[\log \frac{p\left(\boldsymbol{\theta} \mid \mathcal{D}, \mathcal{M}_{j}\right)}{p\left(\boldsymbol{\theta} \mid \mathcal{M}_{j}\right)}\right] \approx \frac{1}{N_{1}} \sum_{k=1}^{N_{1}} \log p\left(\mathcal{D} \mid \boldsymbol{\theta}^{(k)}, \mathcal{M}_{j}\right)-\log p\left(\mathcal{D} \mid \mathcal{M}_{j}\right)
$$

\section{Application of methodology to fatigue test data}

To illustrate the proposed framework, a set of data taken from literature [20] is selected. These data are based on experimental sequences of damage corresponding to sixteen quasi-isotropic glass fiber notched laminates S2-Glass/E733FR, that were subjected to identical and independent tension fatigue tests. See Figure 4 for a graphical representation of the damage series. The tests were conducted under load-controlled fatigue loadings with a frequency of $f=5 \mathrm{~Hz}$, a maximum applied tension of $50 \%$ of their ultimate stress, and a stress ratio $R=0.1$ (relation between the minimum and maximum stress for each cycle).

Some pilot tests revealed that the most suitable value for duty cycle DC for these data is 500 load cycles with a Markov chain assembly of $s=30$ states. Hence, the total number of duty cycles is $N=213900 / 500=428$. In the experiment, some measurements were taken outside of the $[0,1]$ interval because of experimental error in measuring stiffness. Hence, to ensure the existence of an absorbent state for the stochastic process, it is re-defined as the state corresponding to the first measurement that fulfills $\bar{x}_{n_{h}} \geqslant 1$. This definition only affects a small portion of measurements near the absorbent state where transition probabilities are close to value of 1 and hence the likelihood function $p(\mathcal{D} \mid \boldsymbol{\theta}, \mathcal{M})$ is barely affected. 
Four model classes are considered sufficient for the inference, so $N_{M}=4$. This implies that a maximum of 9 parameters are employed for the modeling: from 3 parameters for $\mathcal{M}_{1}$ to 9 for $\mathcal{M}_{4}$ (see Table 1). For each model class, the prior is chosen as the product of independent uniform distributions $^{2}$ for each model parameter, $p\left(\theta_{i} \mid \mathcal{M}_{j}\right)=\mathcal{U}(0,1), i=1, \ldots, d$, where $\boldsymbol{\theta} \in \mathbb{R}^{d}$. Therefore the posterior is given by:

$$
p\left(\boldsymbol{\theta} \mid \mathcal{D}, \mathcal{M}_{j}\right)=c_{2} p\left(\mathcal{D} \mid \boldsymbol{\theta}, \mathcal{M}_{j}\right)
$$

over the unit hypercube of dimension $d$ and it is zero outside of it, where the likelihood function is given by Equation 13. For all test specimens, we consider the fatigue process starting from the no-damage state $\bar{x}_{n_{1}}^{0}$, so the probability $p\left(\bar{x}_{n_{1}}^{0} \mid \boldsymbol{\theta}\right)$ in Equation 13 is equal to 1 .

The M-H algorithm is applied with a multivariate Gaussian for the proposal PDF with identical standard deviation in each dimension, which corresponds to the Random Walk version of the algorithm [55]. See the algorithm configuration in Table 2. CPU times of 1800 [s], $1800[s], 1950[s]$ and $2100[s]$ are required on average to complete the $N_{1}=10^{4}$ simulations for model classes $\mathcal{M}_{1}$ to $\mathcal{M}_{4}$ respectively, using a $2.6 \mathrm{GHz}$ double-core system. Note from Table 2 that the more parameters that are included in the model definition, the smaller the value of $\sigma_{q}$ that is required to achieve an acceptance rate within the recommended interval of $[20 \%-40 \%]$ for a given number of simulations, which agrees with $[43,44]$. It is noted that choosing the first sample of the $\theta_{i}$ and $\theta_{i}^{\prime}$ parameters with values close to the diagonal in Figure 2, reduces significantly the burn-in period and so the time to convergence. Mathematically: $\left(\theta_{1} \approx \theta_{1}^{\prime}\right)<\left(\theta_{2} \approx \theta_{2}^{\prime}\right) \ldots<\left(\theta_{N_{M}} \approx \theta_{N_{M}}^{\prime}\right)$. In fact, this selection for the first sample is consistent with the non-stationarity of the fatigue processes of composite materials, where degradation is not abrupt but gradual in time [56].

The posterior results for the parameters are summarized in Table 3 for models $\mathcal{M}_{1}$ to $\mathcal{M}_{4}$. In Figures 5 to 7 , all two-dimensional projections of the posterior samples are plotted

\footnotetext{
${ }^{2} \mathrm{~A}$ rational way to define a probability model for the prior PDF is to select it such that it produces the largest uncertainty (largest Shannon entropy) [14,36]. The maximum-entropy PDF for a bounded variable is the uniform distribution.
} 
for model classes $\mathcal{M}_{1}$ to $\mathcal{M}_{3}$, respectively. The plot for model class $\mathcal{M}_{4}$ is avoided because its contribution to the inference is negligible and the required space for printing purposes is high. Observing the posterior information for model $\mathcal{M}_{1}$ in Figure 5 , it is noted that $\theta_{1}$ and $\theta_{1}^{\prime}$ are highly correlated along the straight line from $(0,0)$ to $(1,1)$, but they are practically uncorrelated with $p$, which has a narrow range of plausible values between about 0.87 and 0.92 . This means that given our data $\mathcal{D}$, model $\mathcal{M}_{1}$ will produce stochastic damage predictions very close to a stationary model, where the transition probability $p$ is the only model parameter.

\subsection{Assessment of model classes}

In order to choose which model class or set of model classes are more plausible based on data $\mathcal{D}$, the results in Table 3 are not enough. In accordance with the theory in Section 3.3, the best choice among model classes will be those with the higher evidence values, leading to higher posterior probabilities.

In Table 4 the results of the model class assessment are presented for $\mathcal{M}_{j}, j=1, \ldots, 4$ with a uniform prior $p\left(\mathcal{M}_{j} \mid \mathbf{M}\right)=1 / 4$. This model class assessment shows that accounting for the non-stationarity greatly increases the posterior probability of the stochastic models, since the results in Figure 5 show that model $\mathcal{M}_{1}$ is similar to a stationary model with only $p$ as model parameter such as that employed in [20]. Table 4 also shows that $\mathcal{M}_{1}$ and $\mathcal{M}_{4}$ have negligible posterior probability, with the other model classes, $\mathcal{M}_{2}$ and $\mathcal{M}_{3}$, showing the best trade-off between the data and model complexity, leading to posterior probabilities of 0.83 and 0.17 , respectively.

Note also in Table 4 that models $\mathcal{M}_{2}$ to $\mathcal{M}_{4}$ have increasingly higher values of the data fit in comparison with model $\mathcal{M}_{1}$. A possible reason for this can be inferred by observing Figure 8, in which the interpolation curves of unit time transformation are displayed using the MAP parameters values for the interpolation points definition. For models $\mathcal{M}_{2}$ to $\mathcal{M}_{4}$, the first two interpolation points are dedicated to capturing a significant source of nonstationarity of data $\mathcal{D}$ within the first stage of fatigue, leading to a marked improvement in the data fit compared with $\mathcal{M}_{1}$. Models $\mathcal{M}_{3}$ and $\mathcal{M}_{4}$ include additional interpolation points 
that capture the transformation of unit time in the middle-end of the fatigue process, where the modulus reduction data are appreciably dispersed, as shown in Figure 4. This markedly improves the data fit of models $\mathcal{M}_{3}$ and $\mathcal{M}_{4}$. Model $\mathcal{M}_{4}$, which has the most parameters, fits the data the best but also implies less robustness, in the sense of a small variation of model parameters may confer a significant change in the model prediction. This fact is reflected in the increasing value of the EIG.

In addition, the performance of the most probable non-stationary model $\mathcal{M}_{2}$ is compared with a standard non-stationary Bogdanoff and Kozin model (B-K model) [16], denoted $\mathcal{M}_{0}$ here. To account for the non-stationarity of the process when using $\mathcal{M}_{0}$, a quadratic polynomial $n^{\prime}=a n+b n^{2}, \quad(a, b) \in \mathbb{R}$, is used to distort the natural time scale $n$, following the TTCM method by [16]. The posterior PDF of the B-K model parameters (i.e., the one-step transition probability $p$ and polynomial coefficients $a$ and $b$ ) is obtained using the Bayesian methodology proposed in this work. To this end, the M-H algorithm is used with $N=10^{4}$ samples, and lognormal distributions $\ln \mathcal{N}\left(\left(\mu_{a}, \mu_{b}\right), \operatorname{diag}\left(\sigma_{a}^{2}, \sigma_{b}^{2}\right)\right)$ with parameters $\left(\mu_{a}, \mu_{b}\right)=(0,-6)$ and $\left(\sigma_{a}^{2}, \sigma_{b}^{2}\right)=(0.4,0.4)$ are adopted as prior PDFs for model parameters $a$ and $b$, respectively. Then, samples from the posterior for the parameters are further used to simulate sequences of predicted damage from the Markov chain following the standard procedure of conditional sampling [57]. The same procedure to obtain simulated sequences of damage is repeated using posterior samples from model class $\mathcal{M}_{2}$. The posterior means and standard deviations of the predicted damage sequences are shown for $\mathcal{M}_{0}$ and $\mathcal{M}_{2}$ in Figure 9. Note that $\mathcal{M}_{2}$ is able to better reproduce the non-stationarity of the process from the first stage of fatigue damage. We also compute the posterior probabilities of $\mathcal{M}_{0}$ and $\mathcal{M}_{2}$ by computing the log evidence for $\mathcal{M}_{0}$ in the same way as for $\mathcal{M}_{2}$ and find that $P\left(\mathcal{M}_{0} \mid \mathcal{D}, \mathcal{M}_{0} \cup \mathcal{M}_{2}\right)=0.007$ whereas $P\left(\mathcal{M}_{2} \mid \mathcal{D}, \mathcal{M}_{0} \cup \mathcal{M}_{2}\right)=0.993$.

\subsection{Minimum required set of data}

One of the relevant issues when making inference of fatigue models with a dataset based on repeated testing of specimens is to assess the minimum required amount of test specimens to update the model classes and their predictions. From an information point of view, this 
is equivalent to determining the size of the dataset by which the information gain from new test data becomes relative small. This can be done by computing the relative entropy [58] between the posterior from adding the $k^{\text {th }}$ dataset and its prior, which is the posterior based on the previous $(k-1)$ datasets. To this end, let consider the model class $\mathcal{M}_{j}$ with model parameters $\boldsymbol{\theta}$, which are updated with data $\mathcal{D}_{k}$ consisting of $k$ experimental sequences of fatigue-damage: $\left\{\hat{Y}^{(1)}, \ldots, \hat{Y}^{(k)}\right\}, k=1, \ldots, K$, such that $\mathcal{D}_{k-1} \subset \mathcal{D}_{k}$ and $\mathcal{D}_{K} \equiv \mathcal{D}$. The relative entropy (also called cross-entropy and Kullback-Liebler distance) between the posterior PDFs $p\left(\boldsymbol{\theta} \mid \mathcal{D}_{k}, \mathcal{M}_{j}\right)$ and $p\left(\boldsymbol{\theta} \mid \mathcal{D}_{k-1}, \mathcal{M}_{j}\right)$ is defined as:

$$
\int_{\boldsymbol{\Theta}} p\left(\boldsymbol{\theta} \mid \mathcal{D}_{k}, \mathcal{M}_{j}\right) \log _{2}\left[\frac{p\left(\boldsymbol{\theta} \mid \mathcal{D}_{k}, \mathcal{M}_{j}\right)}{p\left(\boldsymbol{\theta} \mid \mathcal{D}_{k-1}, \mathcal{M}_{j}\right)}\right] d \boldsymbol{\theta}
$$

Observe that the relative entropy is the expected information gain (in bits) about $\boldsymbol{\theta}$ from $\mathcal{D}_{k}$ relative to $\mathcal{D}_{k-1}$, so hereinafter we refer to it as the relative information gain (RIG). By the fact that in our framework the posterior $\operatorname{PDF} p\left(\boldsymbol{\theta} \mid \mathcal{D}_{k}, \mathcal{M}_{j}\right)$ is presented by the set of posterior samples $\left\{\theta_{k}^{(t)}\right\}_{t=1}^{N_{1}}$, then the relative information gain of this set can be approximated by ${ }^{3}$ :

$$
\mathrm{RIG} \approx \sum_{t=1}^{N_{1}} \frac{p\left(\mathcal{D}_{k} \mid \theta_{k}^{(t)}, \mathcal{M}_{j}\right) p\left(\theta_{k}^{(t)} \mid \mathcal{M}_{j}\right)}{p\left(\mathcal{D}_{k} \mid \mathcal{M}_{j}\right)} \log _{2}\left[\frac{p\left(\mathcal{D}_{k} \mid \theta_{k}^{(t)}, \mathcal{M}_{j}\right) p\left(\theta_{k}^{(t)} \mid \mathcal{M}_{j}\right) p\left(\mathcal{D}_{k-1} \mid \mathcal{M}_{j}\right)}{p\left(\mathcal{D}_{k-1} \mid \theta_{k-1}^{(t)}, \mathcal{M}_{j}\right) p\left(\theta_{k-1}^{(t)} \mid \mathcal{M}_{j}\right) p\left(\mathcal{D}_{k} \mid \mathcal{M}_{j}\right)}\right]
$$

Notice that in the last equation, the posterior PDFs $p\left(\boldsymbol{\theta} \mid \mathcal{D}_{k}, \mathcal{M}_{j}\right)$ and $p\left(\boldsymbol{\theta} \mid \mathcal{D}_{k-1}, \mathcal{M}_{j}\right)$ are expanded by making substitutions according to Bayes' Theorem.

Figure 10 shows the plots of the cumulative sum of values of RIG from a sequence of specimen fatigue tests for model classes $\mathcal{M}_{1}$ to $\mathcal{M}_{4}$. The numbering of the specimen tests is the actual order in which the experiments were performed. These plots clearly show that there exist a specific size of the dataset, expressed as a number of test specimens, after which the inference does not gain significant information. Based on these results, we choose the value of 11, as the minimum required number of test specimens for adequate inference in our problem, although this value actually depends on each of model classes. In fact, observe that the more complex the model is (in the sense of the number of model parameters, as explained in Section 3.3), the bigger the size of the required dataset, resulting in an optimal

\footnotetext{
${ }^{3}$ The burn-in period must be discarded.
} 
number of specimens from 8 to 11 for model classes $\mathcal{M}_{1}$ to $\mathcal{M}_{4}$, respectively. Notice also that more complex models acquire more information from each test. Both of these experimental observations make sense with the results obtained for the complexity of model classes in the last section.

\subsection{Posterior robust predictive model}

As an alternative to selecting a single model class based on the evidence values, a damage model can be obtained based on the complete set of models $\mathbf{M}$ through the posterior hyper-robust predictive PDF of damage [12]. Based on the Total Probability Theorem, this definition allows us to account for all the probabilistic information in terms of the uncertainty related to both parameters and model class choice for the prediction of damage, as follows:

$$
p\left(x_{n} \mid \mathcal{D}, \mathbf{M}\right)=\sum_{j=1}^{N_{M}} p\left(x_{n} \mid \mathcal{D}, \mathcal{M}_{j}\right) p\left(\mathcal{M}_{j} \mid \mathcal{D}, \mathbf{M}\right)
$$

where $p\left(x_{n} \mid \mathcal{D}, \mathcal{M}_{j}\right)$ is the posterior robust prediction of damage including the parameter uncertainty for model class $\mathcal{M}_{j}$ :

$$
p\left(x_{n} \mid \mathcal{D}, \mathcal{M}_{j}\right)=\int_{\Theta} p\left(x_{n} \mid \boldsymbol{\theta}, \mathcal{D}, \mathcal{M}_{j}\right) p\left(\boldsymbol{\theta} \mid \mathcal{D}, \mathcal{M}_{j}\right) d \boldsymbol{\theta}
$$

If the posterior probabilities $p\left(\mathcal{M}_{j} \mid \mathcal{D}, \mathbf{M}\right)$ given in Table 4 and based on the uniform prior $p\left(\mathcal{M}_{j} \mid \mathbf{M}\right)=1 / 4$ (so that all model classes are considered equally plausible a priori) are substituted into Equation 30, it is clear that the contributions of models $\mathcal{M}_{1}$ and $\mathcal{M}_{4}$ to the hyper-robust predictive model in Equation 30 are negligible, and it can be approximated by:

$$
p\left(x_{n} \mid \mathcal{D}, \mathbf{M}\right) \cong 0.826 p\left(x_{n} \mid \mathcal{D}, \mathcal{M}_{2}\right)+0.174 p\left(x_{n} \mid \mathcal{D}, \mathcal{M}_{3}\right)
$$

The cumulative distribution function $(\mathrm{CDF}) F\left(x_{n} \mid \mathcal{D}, \mathcal{M}\right)$ based on Equation 32 is plotted in Figure 11 where it is compared with the empirical CDF based on the data $\mathcal{D}$, showing good agreement between these data and the posterior predictions based on models $\mathcal{M}_{2}$ and $\mathcal{M}_{3}$ 


\section{Concluding remarks}

A new modeling approach based on Markov chains is proposed to deal with the uncertain physical process and variability of fatigue-based damage in composites. A Bayesian framework is used to quantify and post-process the uncertainty and also to select the optimum model parameterization for fatigue damage modeling. The overall procedure is demonstrated using real data for the evolution of damage for glass-fiber composite coupons subject to tension-tension fatigue.

The following general conclusions are made:

- Accounting for the non-stationarity of the fatigue-damage evolution improves significantly the model predictions;

- Based on the posterior information about a set of model classes, a hyper-robust predictive damage model can be obtained as a multi-model probability density function that gives a higher level of robustness to modeling uncertainty than just taking the most probable model in the set of model classes;

- This Bayesian updating framework can also be used in conjunction with a measure of the information gain from a specimen test to select a minimum set of specimens for damage characterization and prediction, with the ultimate benefit being the avoidance of unnecessary costs in fatigue experimental programs;

- Other phenomena in composites like matrix-crack density, delamination area, etc., that imply cumulative damage processes, can benefit by applying a similar Bayesian framework to Markov chain models of the phenomena.

\section{Acknowledgements}

The two first authors would like to thank the Education Ministry of Spain for the grants FPU 2009-4641, FPU 2009-2390 and GGI3000IDIB; and the California Institute of Technology (Caltech), USA, which kindly hosted them during a part of the course of this 
work. The authors would also like to acknowledge the fruitful discussions with Prof. Michael Ortiz of Caltech and the work of Wei et al. for their valuable set of data published in [20].

The authors also appreciate the reviewers' efforts on the manuscript to provide valuable comments and constructive suggestions. 
[1] S. Carbillet, F. Richard, E. Boubakar, Reliability indicator for layered composites with strongly nonlinear behaviour, Composites Science and Technology 69 (1) (2009) 81-87.

[2] W. Hwang, K. S. Han, Fatigue of composites-Fatigue modulus concept and life prediction, Journal of Composite Materials 20 (2) (1986) 154-165.

[3] S. Abrate, Matrix cracking in laminated composites: a review, Composites Engineering 1 (6) (1991) $337-353$.

[4] A. S. D. Wang, Strength, failure, and fatigue analysis of laminates, in: Engineered Materials Handbook, vol. 1, ASM International, 236-251, 1997.

[5] J. Degrieck, W. Van Paepegem, Fatigue damage modeling of fibre-reinforced composite materials: Review, Applied Mechanics Reviews 54 (4) (2001) 279.

[6] D. An, J.-H. Choi, N. H. Kim, S. Pattabhiraman, Fatigue life prediction based on Bayesian approach to incorporate field data into probability model, Structural Engineering \& Mechanics 37 (4) (2011) $427-442$.

[7] X. Guan, R. Jha, Y. Liu, Model selection, updating, and averaging for probabilistic fatigue damage prognosis, Structural Safety 33 (3) (2011) 242-249.

[8] M. Robinson, M. Crowder, Bayesian methods for a growth-curve degradation model with repeated measures, Lifetime Data Analysis 6 (4) (2000) 357-374.

[9] R. Cross, A. Makeev, E. Armanios, Simultaneous uncertainty quantification of fracture mechanics based life prediction model parameters, International Journal of Fatigue 29 (8) (2007) 1510-1515.

[10] Y. Ling, S. Mahadevan, Integration of structural health monitoring and fatigue damage prognosis, Mechanical Systems and Signal Processing 28 (2012) 89-104.

[11] R. Zhang, S. Mahadevan, Model uncertainty and Bayesian updating in reliability-based inspection, Structural Safety 22 (2000) 145-160.

[12] J. L. Beck, Bayesian system identification based on probability logic, Structural Control and Health Monitoring 17 (7) (2010) 825-847.

[13] R. T. Cox, The Algebra of Probable Inference, The Johns Hopkins University Press, 1961.

[14] E. T. Jaynes, Probability Theory: The Logic of Science, (Ed. G.L. Bretthorst), Cambridge University Press, 2003.

[15] A. Papoulis, Probability, Random Variables, and Stochastic Processes, McGraw Hill, ISBN 0070484481, 1965.

[16] J. L. Bogdanoff, F. Kozin, Probabilistic Models of Cumulative Damage, John Wiley \& Sons, ISBN 0-471-88180-5, 1985.

[17] J. D. Rowatt, P. D. Spanos, Markov chain models for life prediction of composite laminates, Structural Safety 20 (1998) 117-135. 
[18] R. Ganesan, A data-driven stochastic approach to model and analyze test data on fatigue response, Computers \& Structures 76 (4) (2000) 517-531.

[19] Y. Z. Pappas, P. D. Spanos, V. Kostopoulos, Markov chains for damage accumulation of organic and ceramic matrix composites, Journal of Engineering Mechanics 127 (9) (2001) 915-926.

[20] B.-S. Wei, S. Johnson, R. Haj-ali, A stochastic fatigue damage method for composite materials based on Markov chains and infrared thermography, International Journal of Fatigue 32 (2010) 350-360.

[21] S. Johnson, B. Wei, R. Haj-Ali, A stochastic fatigue damage model for composite single lap shear joints based on Markov chains and thermoelastic stress analysis, Fatigue \& Fracture of Engineering Materials \& Structures 33 (12) (2010) 897-910.

[22] Y. Lin, J. Yang, A stochastic theory of fatigue crack propagation, AIAA journal 23 (1) (1985) $117-124$.

[23] W.-F. Wu, On the Markov approximation of fatigue crack growth, Probabilistic Engineering Mechanics 1 (4) (1986) 224-233.

[24] K. Sobczyk, B. Spencer, Random Fatigue: From Data to Theory, Academic Press, 1992.

[25] J. A. Bea, M. Doblaré, L. Gracia, Evaluation of the probability distribution of crack propagation life in metal fatigue by means of probabilistic finite element method and B-models, Engineering Fracture Mechanics 63 (6) (1999) 675-711.

[26] W. Wu, C. Ni, Probabilistic models of fatigue crack propagation and their experimental verification, Probabilistic Engineering Mechanics 19 (3) (2004) 247-257.

[27] M. Giorgio, M. Guida, G. Pulcini, An age- and state-dependent Markov model for degradation processes, IIE Transactions 43 (2011) 621-632.

[28] M. Guida, G. Pulcini, A continuous-state Markov model for age- and state-dependent degradation processes, Structural Safety 33 (6) (2011) 354-366.

[29] K. Sobczyk, Stochastic models for fatigue damage of materials, Advances in Applied Probability 19 (3) (1987) 652-673.

[30] B. F. Spencer Jr, J. Tang, Markov process model for fatigue crack growth, Journal of Engineering Mechanics 114 (12) (1988) 2134-2157.

[31] F. Casciati, P. Colombi, L. Faravelli, Fatigue crack size probability distribution via a filter technique, Fatigue \& Fracture of Engineering Materials \& Structures 15 (5) (1992) 463-475.

[32] J. Lemaitre, J. Cordebois, J. Dufailly, Sur le couplage endommagement-elasticit, Comptes rendus de l'Acadmie des Sciences, Paris, B. (1979) 391.

[33] G. Z. Voyiadjis, P. I. Kattan, A comparative study of damage variables in continuum damage mechanics, International Journal of Damage Mechanics 18 (4) (2009) 315-340.

[34] F. N. Fritsch, R. E. Carlson, Monotone piecewise cubic interpolation, SIAM Journal on Numerical Analysis 17 (2) (1980) 238-246. 
[35] R. T. Cox, Probability, frequency and reasonable expectation, American Journal of Physics (17) (1946) $1-13$.

[36] E. T. Jaynes, Papers on Probability, Statistics and Statistical Physics, (Ed. R.D. Rosenkrantz), Kluwer Academic Publishers, 1983.

[37] A. Tarantola, Inverse Problem Theory and Methods for Model Parameters Estimation, SIAM, 2005.

[38] P. Whittle, Some distribution and moment formulae for the Markov chain, Journal of the Royal Statistical Society. Series B (Methodological) (1955) 235-242.

[39] P. Billingsley, Statistical methods in Markov chains, The Annals of Mathematical Statistics 32 (1) (1961) 12-40.

[40] F. Liang, C. Liu, J. Chuanhai, Advanced Markov Chain Monte Carlo Methods, Wiley Online Library, 2010.

[41] N. Metropolis, A. W. Rosenbluth, M. N. Rosenbluth, A. H. Teller, E. Teller, Equation of state calculations by fast computing machines, The Journal of Chemical Physics 21 (1953) 1087-1092.

[42] W. K. Hastings, Monte Carlo sampling methods using Markov chains and their applications, Biometrika 57 (1) (1970) 97-109.

[43] A. Gelman, G. Roberts, W. Gilks, Efficient Metropolis jumping rules, Bayesian Statistics 5 (1996) 599-608.

[44] G. Roberts, J. Rosenthal, Optimal scaling for various Metropolis-Hastings algorithms, Statistical Science 16 (4) (2001) 351-367.

[45] J. Hoeting, D. Madigan, A. Raftery, C. Volinsky, Bayesian model averaging: A tutorial, Statistical Science (1999) 382-401.

[46] J. L. Beck, K. V. Yuen, Model selection using response measurements: Bayesian probabilistic approach, Journal of Engineering Mechanics 130 (2004) 192.

[47] A. E. Raftery, D. Madigan, J. A. Hoeting, Bayesian model averaging for linear regression models, Journal of the American Statistical Association (1997) 179-191.

[48] S. F. Gull, Developments in maximum-entropy data analysis, Maximum Entropy and Bayesian Methods (1989) 53-71.

[49] J. L. Beck, L. S. Katafygiotis, Updating models and their uncertainties. I: Bayesian statistical framework, Journal of Engineering Mechanics 124 (4) (1998) 455-462.

[50] L. S. Katafygiotis, H. F. Lam, Tangential projection algorithm for manifold representation in unidentifiable model updating problems, Earthquake Engineering \& Structural Dynamics 31 (4) (2002) 791-812.

[51] C. Papadimitriou, J. L. Beck, L. S. Katafygiotis, Asymptotic expansions for reliability and moments of uncertain systems, Journal of Engineering Mechanics 123 (12) (1997) 1219-1229.

[52] M. Muto, J. L. Beck, Bayesian updating and model class selection for hysteretic structural models using 
stochastic simulation, Journal of Vibration and Control 14 (1-2) (2008) 7-34.

[53] A. E. Gelfand, D. K. Dey, Bayesian model choice: asymptotics and exact calculations, Journal of the Royal Statistical Society. Series B (Methodological) 56 (3) (1994) 501-514.

[54] S. H. Cheung, J. L. Beck, Calculation of posterior probabilities for Bayesian model class assessment and averaging from posterior samples based on dynamic system data, Computer-Aided Civil and Infrastructure Engineering 25 (5) (2010) 304-321.

[55] W. R. Gilks, S. Richardson, D. J. Spiegelhalter, Markov Chain Monte Carlo in Practice, Chapman and Hall, 1996.

[56] R. Talreja, A continuum mechanics characterization of damage in composite materials, Proc. R. Soc. London 399 (1985) 195-216.

[57] R. Y. Rubinstein, D. P. Kroese, Simulation and the Monte Carlo Method., John Wiley \& Sons, Hoboken, New Jersey, second edn., 2008.

[58] S. Kullback, R. A. Leibler, On Information and Sufficiency, The Annals of Mathematical Statistics 22 (1) (1951) 79-86. 
STOCHASTIC MODELING

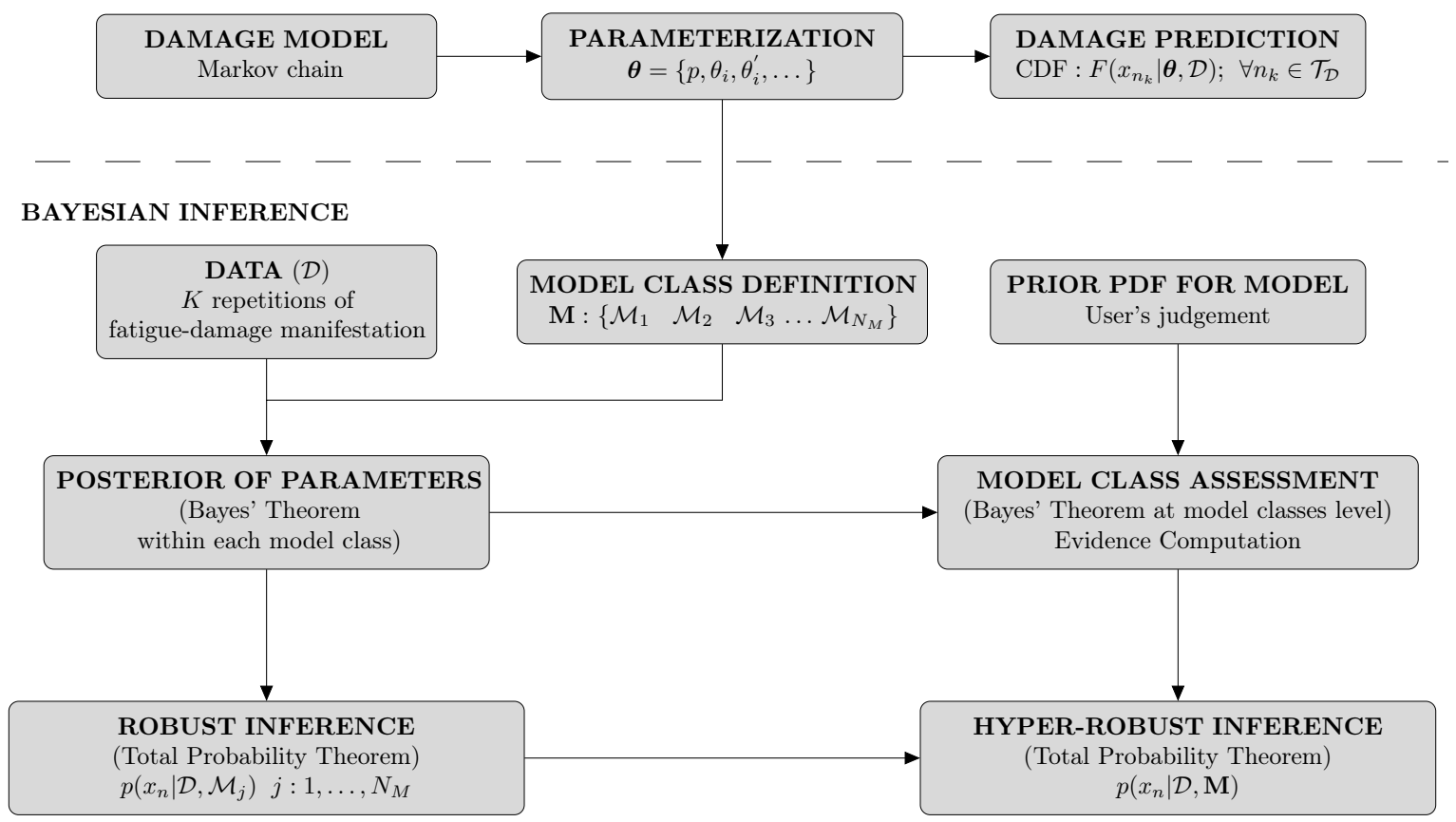

Figure 1: Bayesian framework for fatigue-based damage prediction. 


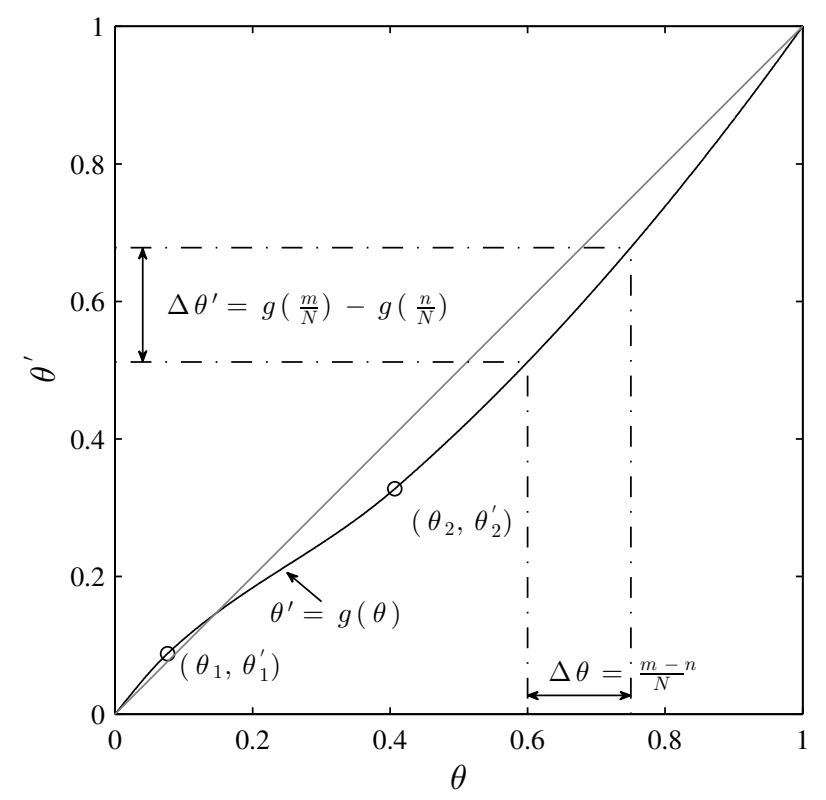

Figure 2: Spline transformation $\theta^{\prime}=g(\theta)$ of the unit time scale $\theta$ controlled by the set of interpolation points $\left\{(0,0),\left(\theta_{1}, \theta_{1}^{\prime}\right), \ldots\left(\theta_{j}, \theta_{j}^{\prime}\right),(1,1)\right\} ; \theta_{j}>\theta_{j-1}$ and $\theta_{j}^{\prime}>\theta_{j-1}^{\prime} ; j>1$. The grey line would correspond to a stationary process in which the time transformation does not take place, so $\theta^{\prime}=\theta$; whereas the darker curve represents the proposed non-stationary scheme.

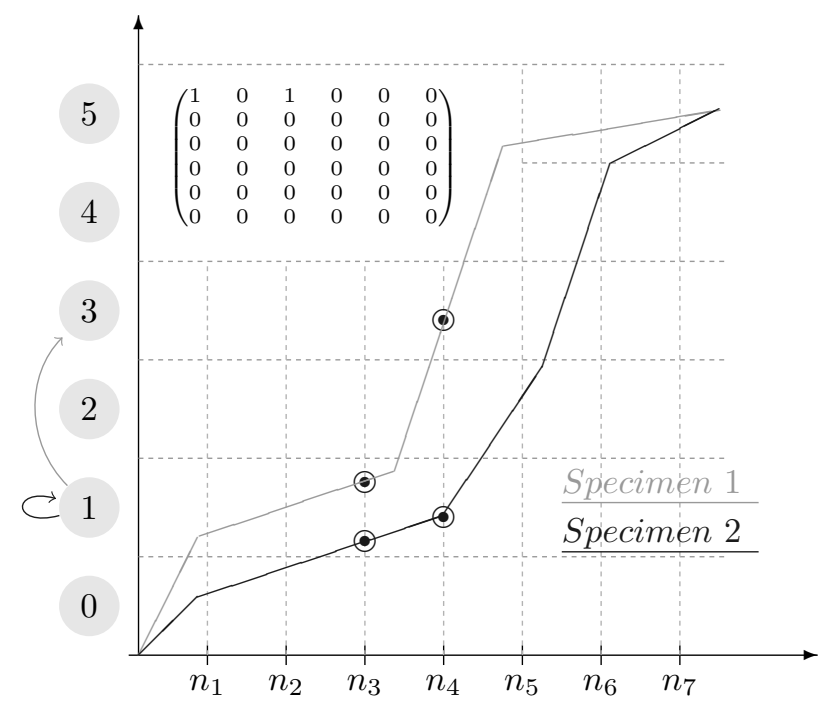

Figure 3: Toy example for obtaining $\mathbf{f}_{n=n_{4}}$ when a Markov chain with 6-states is studied with data from two specimens. The damage states are represented on the vertical axis. The set of DC are represented on the horizontal axis. Observe that a transition between states 1 (for $n=n_{3}$ ) and 3 (for $n=n_{4}$ ) occurs in specimen 1 , so $f_{n=n_{4}}^{1,3}=1$. Notice that in specimen 2, damage remains in the same state for $n=n_{3}$ and $n=n_{4}$, so $f_{n=n_{4}}^{1,1}=1$. See the example transition count matrix $\mathbf{f}_{n=n_{4}}$ in the upper-left side of this figure. 


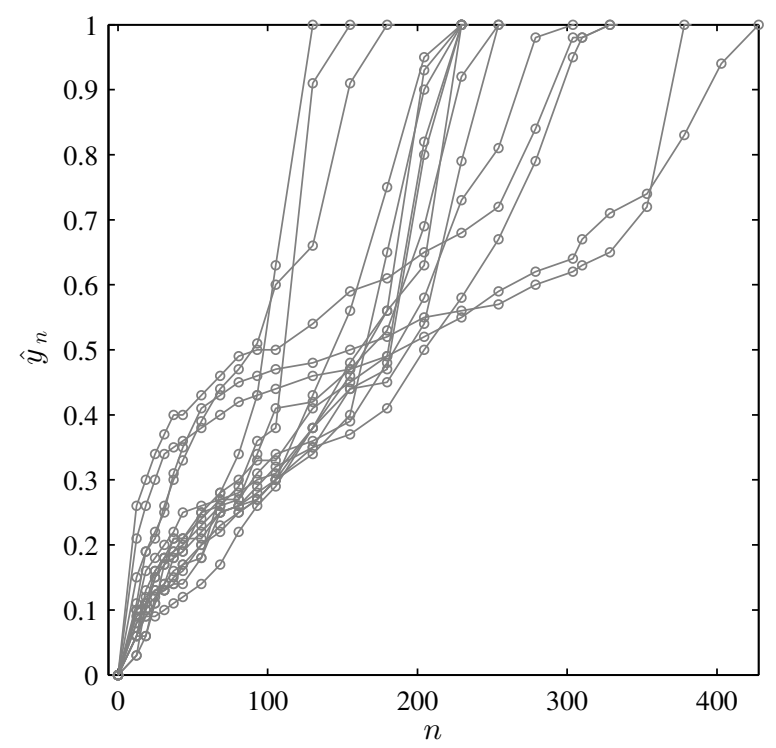

Figure 4: Experimental sequences of damage for quasi-isotropic notched S2-Glass/E733FR laminates taken from $[20]$.

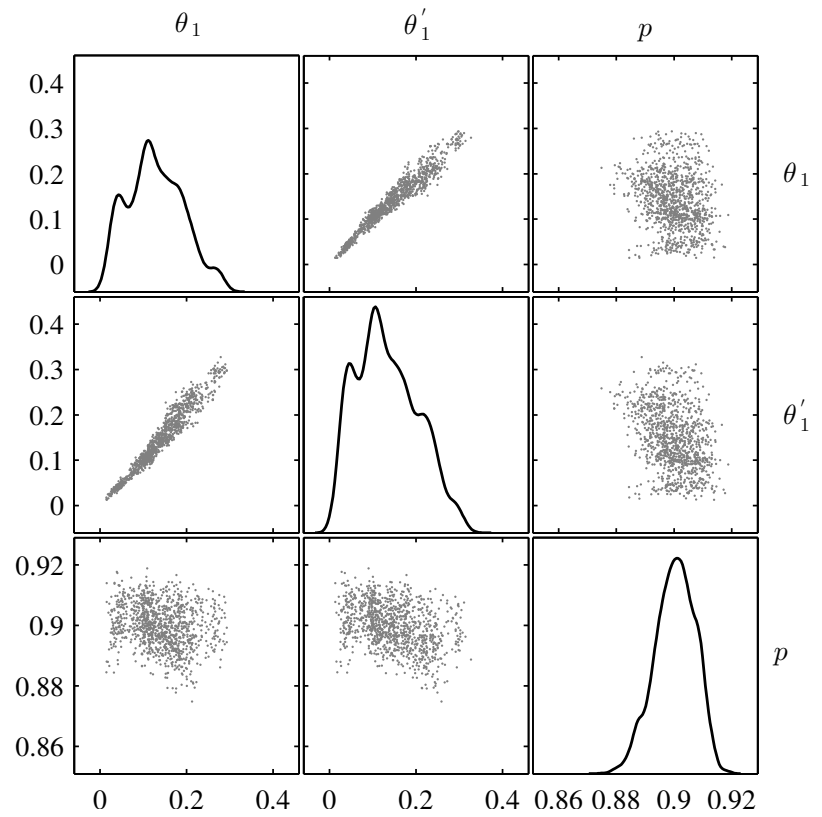

Figure 5: Plots of $10^{4}$ posterior samples in the $\boldsymbol{\theta}$ space when updating model class $\mathcal{M}_{1}$ with fatigue data $\mathcal{D}$ [20]. On the diagonal, kernel density estimates are shown for the marginal posterior PDFs of the respective parameters. 


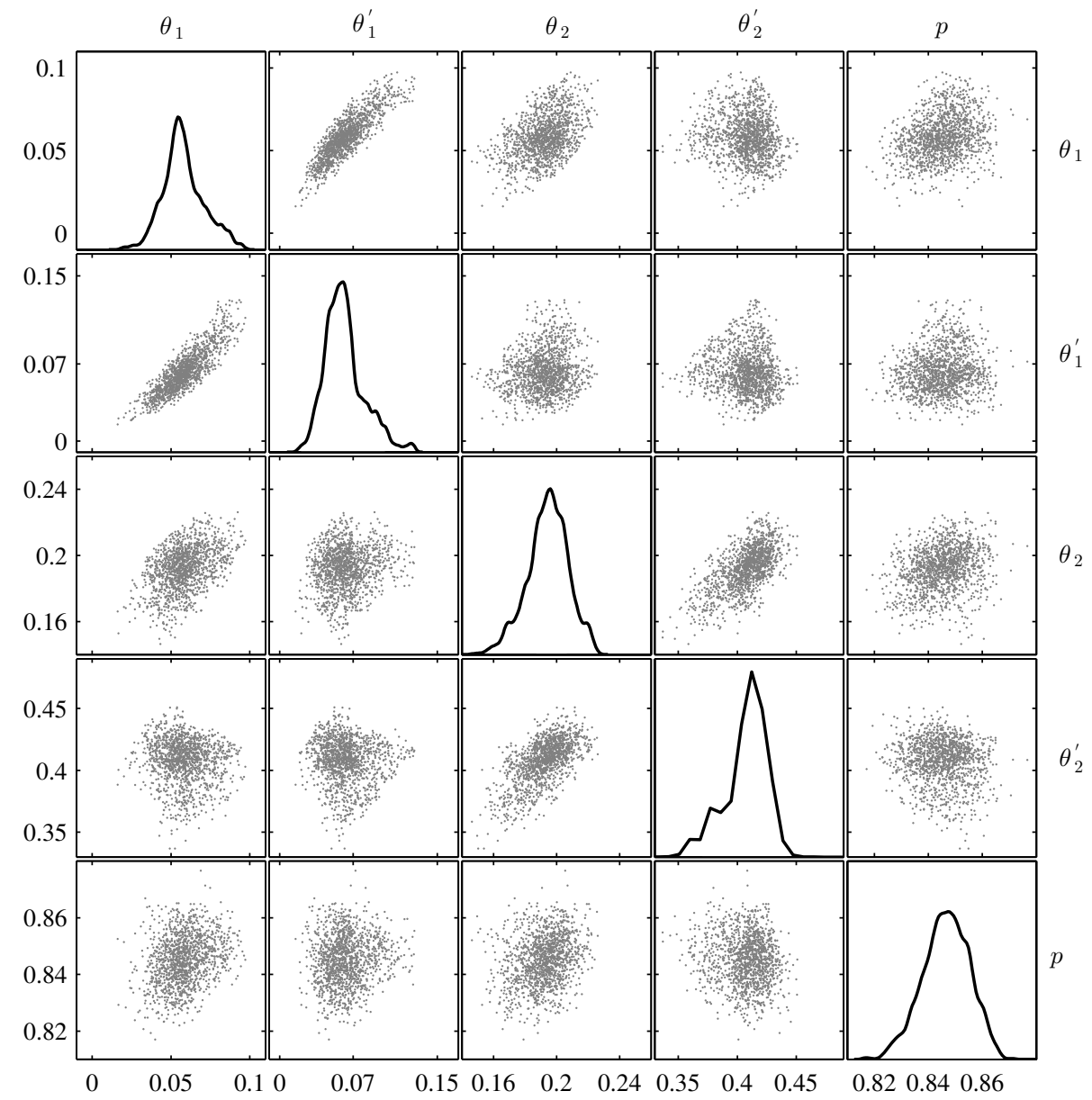

Figure 6: Plots of $10^{4}$ posterior samples in the $\boldsymbol{\theta}$ space when updating model class $\mathcal{M}_{2}$ with fatigue data $\mathcal{D}$ [20]. On the diagonal, kernel density estimates are shown for the marginal posterior PDFs of the respective parameters. 


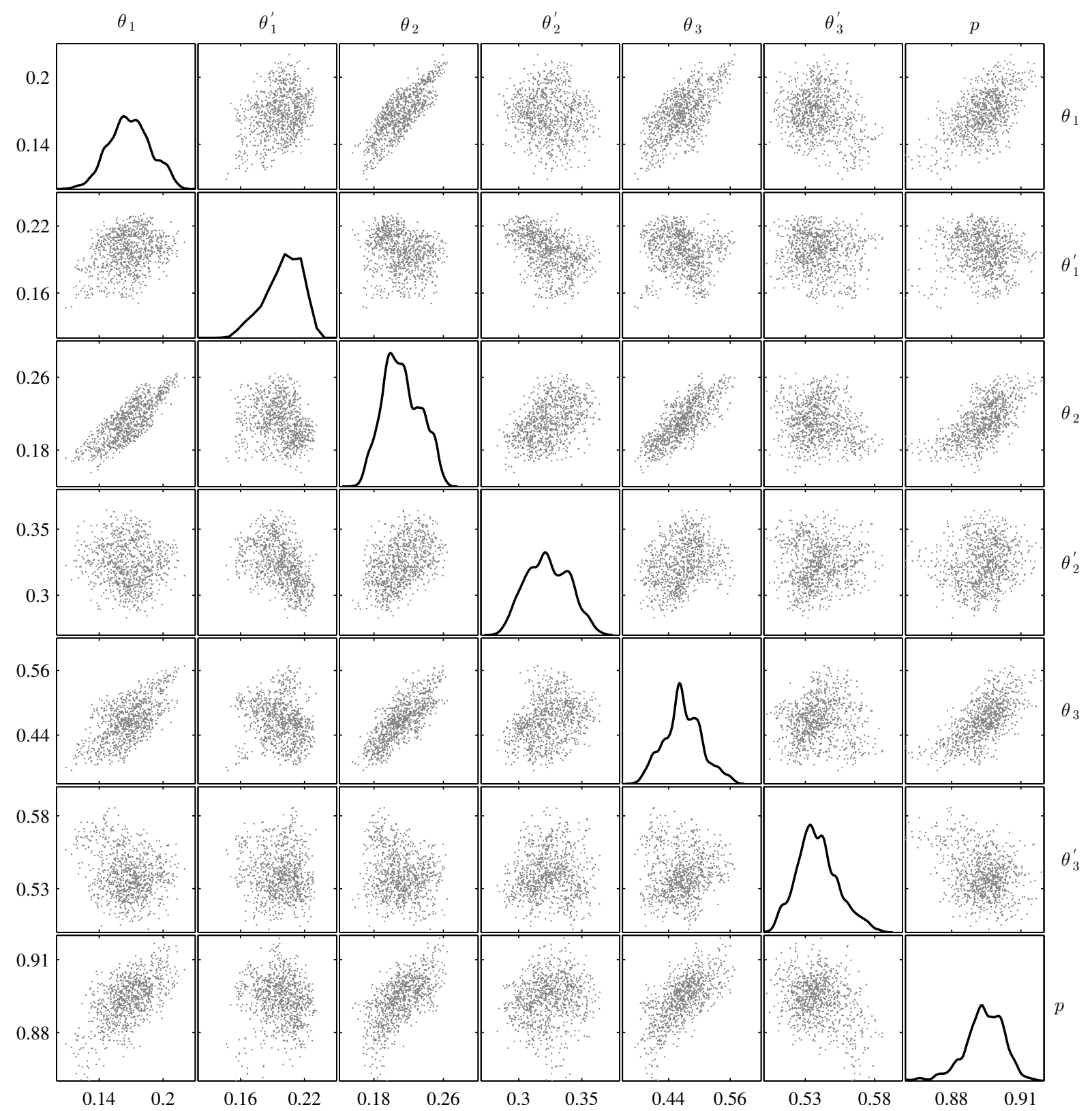

Figure 7: Plots of $10^{4}$ posterior samples in the $\boldsymbol{\theta}$ space when updating model class $\mathcal{M}_{3}$ with fatigue data $\mathcal{D}$ [20]. On the diagonal, kernel density estimates are shown for the marginal posterior PDFs of the respective parameters. 


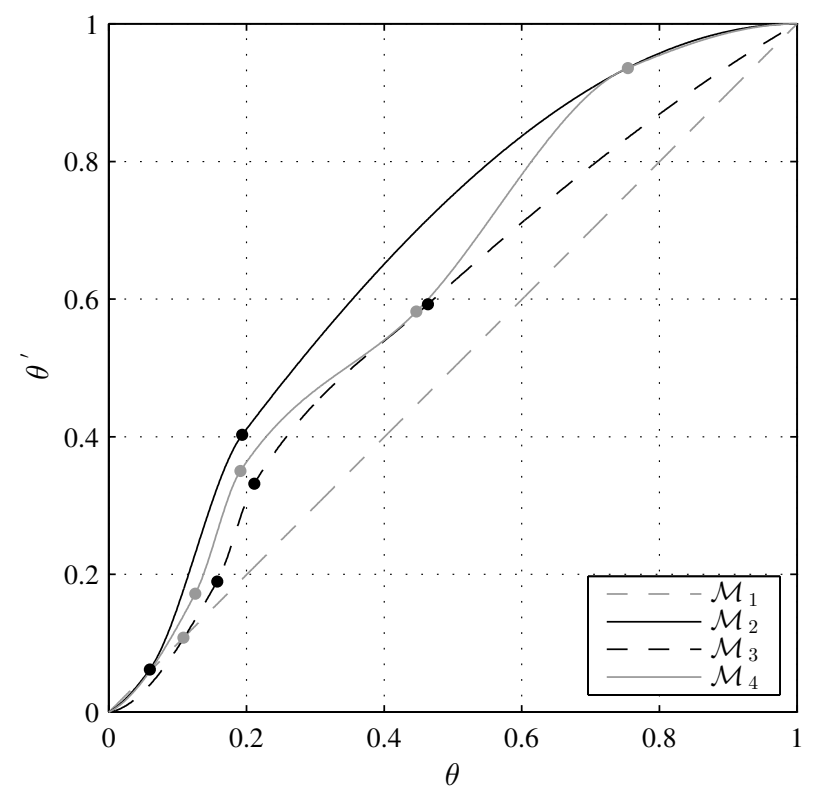

Figure 8: Plots of interpolation curves of unit time transformation for model classes $\mathcal{M}_{1}$ to $\mathcal{M}_{4}$. The interpolation points are the MAP values of $\left(\theta_{i}, \theta_{i}^{\prime}\right)$, which are drawn from left to right in increasing order within their respective curve.

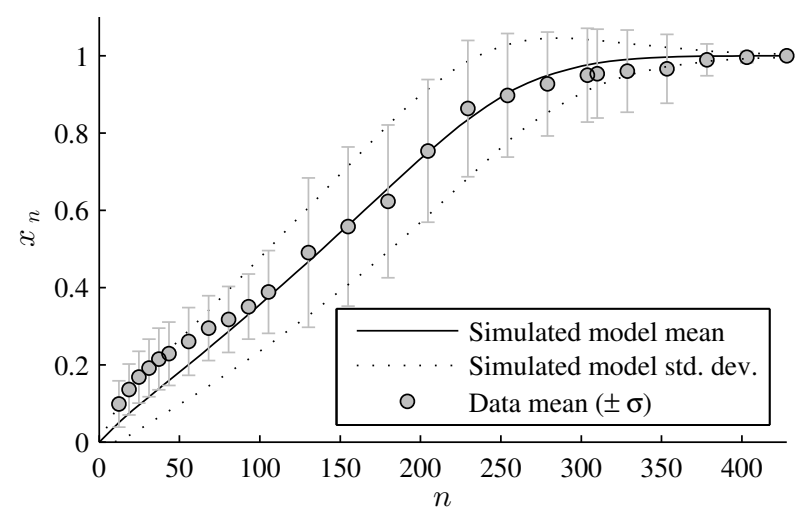

(a) B-K model

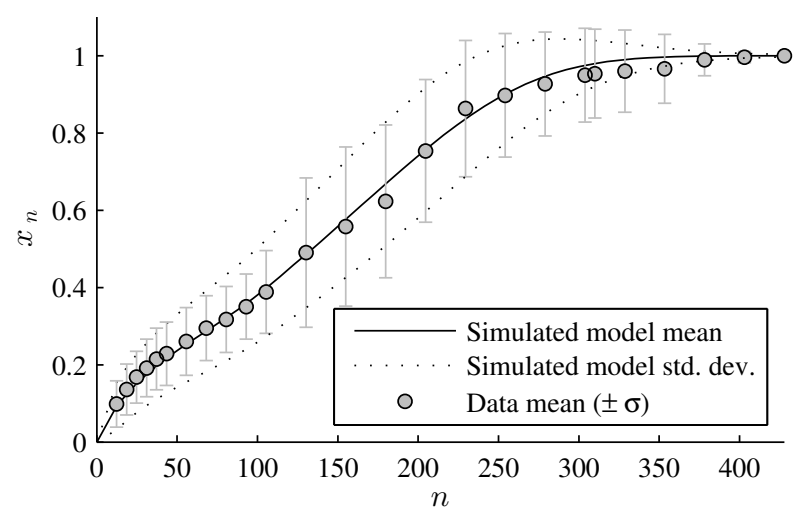

(b) $\mathcal{M}_{2}$

Figure 9: Monte Carlo mean and standard deviation of simulated sequences of damage by using a B-K model (left) and model class $\mathcal{M}_{2}$ (right), in comparison with the mean and standard deviation of experimental sequences. 


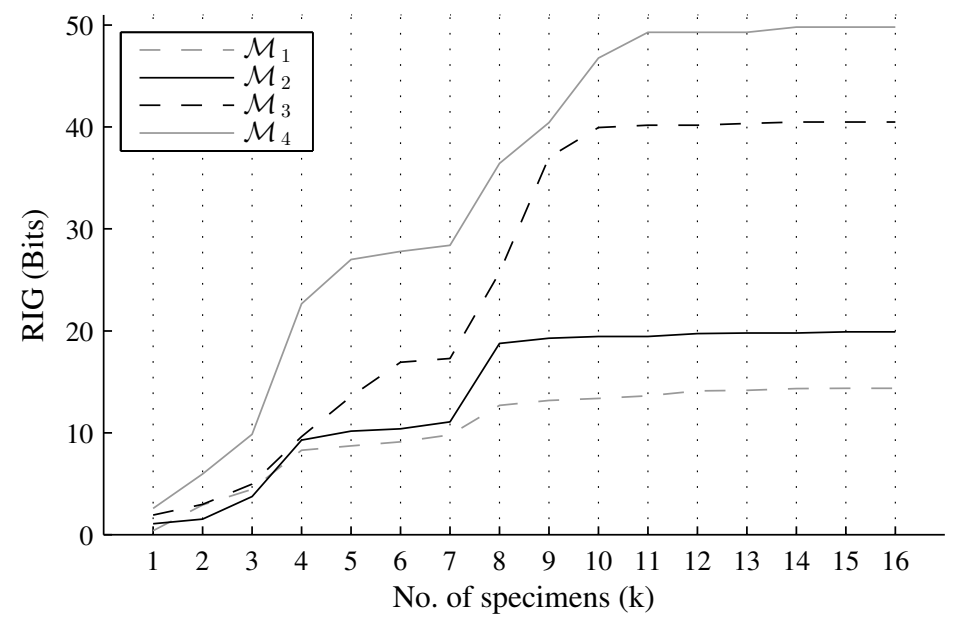

Figure 10: Plots of the cumulative sum of values of the RIG between consecutive posteriors $p\left(\boldsymbol{\theta} \mid \mathcal{D}_{k}, \mathcal{M}_{j}\right)$, $k=1, \ldots, K$, for models classes $\mathcal{M}_{1}$ to $\mathcal{M}_{4}$. When $k=1$, the RIG about $\boldsymbol{\theta}$ is computed from $\mathcal{D}_{1}$ relative to the prior PDF. 

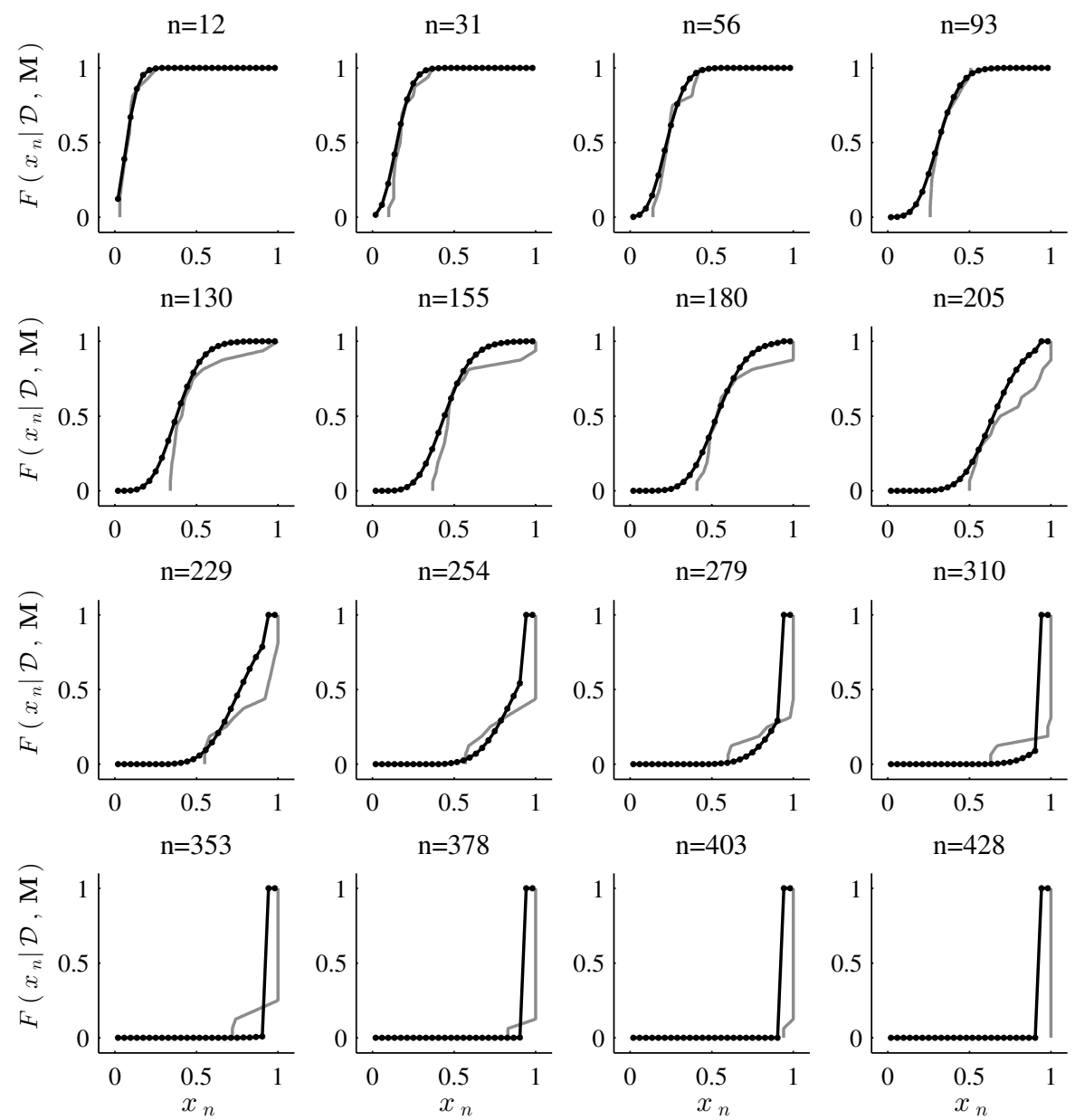

Figure 11: Plots of CDF of the posterior hyper-robust predictive damage model for 16 duty cycles covering the full fatigue time (dark dotted curves). The empirical CDF of damage based on the same data $\mathcal{D}$ from [20], are given as the grey solid curves. 


\begin{tabular}{llllllllll}
\hline Model Class & \multicolumn{10}{c}{ Parameters } \\
\hline $\mathcal{M}_{1}$ & $\theta_{1}$ & $\theta_{1}^{\prime}$ & - & - & - & - & - & $p$ \\
$\mathcal{M}_{2}$ & $\theta_{1}$ & $\theta_{1}^{\prime}$ & $\theta_{2}$ & $\theta_{2}^{\prime}$ & - & - & - & $p$ \\
$\vdots$ & & & & & $\ddots$ & & & \\
$\mathcal{M}_{N_{M}}$ & $\theta_{1}$ & $\theta_{1}^{\prime}$ & $\theta_{2}$ & $\theta_{2}^{\prime}$ & $\ldots$ & $\theta_{N_{M}}$ & $\theta_{N_{M}}^{\prime}$ & $p$ \\
\hline
\end{tabular}

Table 1: Parameterization scheme for the set $\mathbf{M}$ of $N_{m}$ model classes

\begin{tabular}{lcccr}
\hline & $\mathcal{M}_{1}$ & $\mathcal{M}_{2}$ & $\mathcal{M}_{3}$ & $\mathcal{M}_{4}$ \\
\hline$\sigma_{q}$ & 0.007 & 0.005 & 0.005 & 0.0035 \\
$N_{1}$ & $10^{4}$ & $10^{4}$ & $10^{4}$ & $10^{4}$ \\
Burn-in & 400 & 400 & 500 & 700 \\
Acc-rate & $23 \%$ & $34 \%$ & $37 \%$ & $40 \%$ \\
\hline
\end{tabular}

Table 2: Metropolis-Hastings algorithm configuration together with results of the acceptance rate for the stochastic simulation used for inferring models $\mathcal{M}_{1}$ to $\mathcal{M}_{4}$.

\begin{tabular}{|c|c|c|c|c|c|c|c|c|c|c|}
\hline & & $\theta_{1}$ & $\theta_{1}^{\prime}$ & $\theta_{2}$ & $\theta_{2}^{\prime}$ & $\theta_{3}$ & $\theta_{3}^{\prime}$ & $\theta_{4}$ & $\theta_{4}^{\prime}$ & $p$ \\
\hline \multirow{3}{*}{$\mathcal{M}_{1}$} & MAP & 0.1083 & 0.1080 & - & - & - & - & - & - & 0.89 \\
\hline & c.o.v. $(\%)$ & 45.31 & 48.76 & - & - & - & - & - & - & 0.75 \\
\hline & std & 0.0514 & 0.0569 & - & - & - & - & - & - & 0.0068 \\
\hline \multirow{3}{*}{$\mathcal{M}_{2}$} & MAP & 0.0593 & 0.0618 & 0.2042 & 0.4207 & - & - & - & - & 0.8516 \\
\hline & c.o.v. $(\%)$ & 28.67 & 31.54 & 09.84 & 05.27 & - & - & - & - & 0.13 \\
\hline & std & 0.0167 & 0.0213 & 0.0194 & 0.0223 & - & - & - & - & 0.0110 \\
\hline \multirow{3}{*}{$\mathcal{M}_{3}$} & MAP & 0.1573 & 0.1898 & 0.2051 & 0.3212 & 0.4587 & 0.5454 & - & - & 0.89 \\
\hline & c.o.v. $(\%)$ & 13.64 & 13.73 & 09.65 & 04.91 & 09.36 & 03.22 & - & - & 0.97 \\
\hline & std & 0.0219 & 0.0264 & 0.0202 & 0.0161 & 0.0428 & 0.0174 & - & - & 0.0087 \\
\hline \multirow{3}{*}{$\mathcal{M}_{4}$} & MAP & 0.1254 & 0.1719 & 0.1652 & 0.3715 & 0.4131 & 0.5941 & 0.7137 & 0.9353 & 0.8762 \\
\hline & c.o.v. $(\%)$ & 30.06 & 33.05 & 10.62 & 05.07 & 07.27 & 05.80 & 10.40 & 01.61 & 02.09 \\
\hline & std & 0.0312 & 0.0449 & 0.0188 & 0.0173 & 0.0308 & 0.0348 & 0.0721 & 0.0152 & 0.0180 \\
\hline
\end{tabular}

Table 3: Posterior results for model parameters. MAP means maximum a posteriori estimate and corresponds to the parameter value that maximize the probability $p(\boldsymbol{\theta} \mid \mathcal{D}, \mathcal{M})$. c.o.v. is the posterior coefficient of variation and std is the posterior standard deviation of each parameter. 


\begin{tabular}{lcccc}
\hline & Log evidence & Datafit & EIG & Posterior Probability \\
\hline $\mathcal{M}_{1}$ & -183.43 & -180.31 & 3.11 & $3.5 \cdot 10^{-18}$ \\
$\mathcal{M}_{2}$ & -166.13 & -161.61 & 4.52 & 0.826 \\
$\mathcal{M}_{3}$ & -166.73 & -157.05 & 9.68 & 0.174 \\
$\mathcal{M}_{4}$ & -170.73 & -156.25 & 14.47 & $1.74 \cdot 10^{-5}$ \\
\hline
\end{tabular}

Table 4: Results of the terms in Equation 17 for each model class. The 2 nd column is the difference of the next two columns (EIG= Expected Information Gain). The 5th column is the probability of each model class within the set of candidates $\mathbf{M}$, conditional on the data $\mathcal{D}$ and choosing a uniform prior $p\left(\mathcal{M}_{j} \mid \mathbf{M}\right)=1 / 4$. 